\title{
A Virtual Crack Extension Method to Compute Energy Release Rates using a Complex- valued Finite Element Method
}

\author{
H. Millwater, D. Wagner, A. Baines, A. Montoya \\ University of Texas at San Antonio
}

\begin{abstract}
The complex-valued finite element method, ZFEM, is proposed as a new virtual crack extension method to compute the energy release rate. The energy release rate is computed as a numerical derivative of the strain energy with respect to a crack extension using the complex Taylor series expansion method (CTSE). This is accomplished using a finite element method with complex nodal coordinates and extending the crack by a very small quantity along the imaginary directions of the complex nodal coordinates. The resulting finite element formulation becomes complex valued with the imaginary component of the strain energy containing the energy release rate. This method retains the conceptual simplicity of numerical differentiation but eliminates numerical issues regarding perturbation of the crack size. Both two- and three- dimensional examples are provided. Numerical examples indicate that the energy release rate computed using ZFEM is of the same accuracy as the domain-based $\mathrm{J}$ integral formulation. The method is very general and can be applied to self-similar or non-self-similar crack extensions.
\end{abstract}

Keywords: strain energy release rate; virtual crack extension method; stiffness derivative method; complex variable finite element method; complex Taylor series expansion method

\section{Introduction and Background}

The calculation of the energy release rate, $G= \pm d U / d A$, is a fundamental tenet of fracture mechanics and is necessary for structural integrity assessments and fracture control plans. As a result, significant attention has been allocated towards accurate numerical methods to calculate the energy release rate, particularly with regards to the finite element implementation.

Researchers realized early on that the mathematics of the energy release rate implied a virtual crack extension (VCE) method. Historically several finite element-based approaches have 
been explored such as: independent analyses at discrete crack sizes increments [Watwood, 1969; Anderson et. al, 1971], various approaches to the stiffness derivative method [Parks, 1974; BanksSills and Sherman, 1992; Hellen, 1975; Suo and Combescure, 1992; Giner et al., 2002; Yang, et. al., 2001; Waisman, 2010], and variational approaches [Barbero and Reddy, 1990; Haber \& Ko, 1985; Lin \& Abel, 1988; Hwang et al., 1998]. Ingraffea and Wawrzynek provide an overview of various methods [Ingraffea and P.A. Wawrzynek, 2003]

The simplest approach is to employ a finite difference formula and estimate the energy release rate as $G \approx-(U(A+\Delta A)-U(A) / \Delta A)$, where $U(a)$ and $U(A+\Delta A)$ are the strain energies obtained from two separate finite element analyses with the crack slightly extended in the second analysis. Other formulas such as central differencing may be used. This approach was employed by Watwood to estimate the energy release rate from a center- and side-cracked panels by using multiple independent finite element analyses with a series of small crack extensions [Watwood, 1969]. Errors of approximately $2 \%$ of the mode I stress intensity factor were obtained. Similarly, Anderson et. al considered a center crack panel and used central differencing to estimate $G$. While the simplicity of the approach is appealing, numerical differentiation using a finite differencing formula is problematic as the crack extension, or series of crack extensions, $\Delta A$, must be carefully selected to obtain an accurate result, and careful alignment of the meshes is required. The issue of selecting a suitable step size is a well-known deficiency of the finite difference method.

For the stiffness derivative approach, the derivative of the strain energy was incorporated into the finite element equations as a derivative of the stiffness matrix with respect to a local crack extension. A finite difference operation was initially employed to obtain an approximation to $d K / d a$, e.g., $d K / d A \approx(K(A+\Delta A)-K(A)) / \Delta A$, resulting in a numerical implementation that was efficient but dependent on the employed crack size extension [Parks, 1974; Hellen, 1975]. Subsequently, direct differentiation methods were implemented such that the stiffness derivative was computed analytically thereby circumventing the numerical differentiation issues with the crack extension size [Waisman, 2010; Giner et al., 2002]. Banks-Sills and Sherman proved that the 
VCE-stiffness-derivative and $\mathrm{J}$ integral methods reduce to identical expressions and obtain nearidentical numerical results [Banks-Sills and Sherman, 1992]. Extension of VCE methods to $2^{\text {nd }}$ order derivatives has been accomplished by Suo and Combescure using the stiffness derivative method [Suo and Combescure, 1992]. Second order derivatives are needed for stability analysis, and multi-cracked systems.

A fundamental improvement that removed the step size issue was introduced by Haber and Ko through the development of a variational approach to obtain the required numerical derivatives. [Habor \& Ko, 1985] This method obviates the need to select a perturbed crack size. Subsequent implementations and enhancements were obtained by Lin and Abel, and Babero and Reddy [Lin \& Abel, 1988; Barbero and Reddy, 1990]. Hwang et al. extended the method to second order derivatives [Hwang, 1998]. In the case of multiple crack systems, for example, the variation of energy release rate at one crack tip due to the growth of any other crack must be calculated to determine the strength of the interaction. In all these cases, the energy release rate was determined in a single finite element analysis.

The generally considered state-of-the-art $\mathrm{J}$ integral method is the domain-based integral [Shih, 1986]. Solid mechanics finite element codes contain automatic or user-defined closedcontour paths around the crack tip from which multiple J-integral results are computed. A consistent, repeated value from multiple paths is generally chosen as the numerical value of the Jintegral.

While the $\mathrm{J}$ integral has shown outstanding success, it must be modified to analyze different physical phenomena. As examples, variations to the J-integral include: residual stresses [Lei et al., 2000], path independent J-integral for incremental plasticity [Carpenter et al., 1986], path independent J-integrals for thermo-mechanical problems [Wilson and Yu, 1979], path independent J-integral for elasto dynamic crack propagation [Nishioka et al., 1988], path independent integral for time-dependent creep behavior in a quasi-static step, known as the $C_{t}$-integral [Riedel, 1981], 
among others. Hence, the derivation and implementation of the $\mathrm{J}$ integral for complex material models are non-trivial.

In this paper, a new VCE method is presented for computing the strain energy release rate based upon the complex-variable finite element method ZFEM. ZFEM numerically differentiates the finite element system of equations with respect to an extension of the crack length or crack area using the complex Taylor series expansion method (CTSE) [Squire and Trapp, 1998]. In concept, two analyses are computed with a slight perturbation in the crack length or crack area and the change in the strain energy is computed. However, using CTSE, these computations are accomplished in one complex finite element analysis and the energy release rate is contained within the imaginary component of the strain energy. The significant feature of CTSE is that step size issues are non-existent if a sufficiently small step size is used.

CTSE is a general numerical differentiation method that, in contrast to the finite difference method, perturbs the parameter of interest along the imaginary axis. That is, variable $X=x_{0}$ is made complex and is perturbed to $X=x_{0}+i h$, where $i$ denotes an imaginary number and $h$ denotes the step size. The formula for the derivatives can be derived from the Taylor series representation of the function evaluated at the complex sample point,

$$
f\left(x_{0}+i h\right)=f\left(x_{0}\right)+f^{(1)}\left(x_{0}\right) \frac{i h}{1 !}+f^{(2)}\left(x_{0}\right) \frac{(i h)^{2}}{2 !}+f^{(3)}\left(x_{0}\right) \frac{(i h)^{3}}{3 !}+\text { H.O.T. }
$$

where $f^{(1)}$ denotes the first derivative, $f^{(2)}$ the second, etc. Taking the imaginary part of both sides of Eq. (1), solving for the first derivative, and ignoring terms of $O\left(h^{2}\right)$ yields an estimate of the first derivative.

$$
f^{(1)}\left(x_{o}\right) \approx \frac{\operatorname{Im}\left(f\left(x_{o}+i h\right)\right)}{h}
$$

Note that no difference operation is needed for the first derivative. This means that the step size $h$ can be made arbitrarily small with no concern about round-off error. In practice, $h$ is very small, typically $10^{-10}$ times the size of the element at the crack tip. Note that if $h$ is very small, higher order effects of $O\left(h^{2}\right)$ can be made negligible. 
Implementation into a solid mechanics finite element code was first described in Voorhees et al. as a general shape sensitivity method [Voorhees, et al., 2011]. CTSE was used in a fatigue analysis to compute sensitivities of the cycles-to-failure with respect to fatigue parameters [Voorhees, et al., 2012]. More specific to fracture mechanics, CTSE was used to compute very accurate weight functions by computing the derivative of the crack opening displacement with respect to crack length and the stress intensity factor [Wagner and Millwater, 2012; Millwater, et al., 2013]. Other examples using the complex finite element method for weight function development include [Zhao, $\mathrm{Wu}$ and Tong, 2015; Jing and $\mathrm{Wu}$; 2015]. ZFEM has been extended to nonlinear problems in plasticity [Montoya et. al, 2014] and creep [Gomez-Farias et. al, 2015]. Examples in other disciplines include: aerodynamics [Anderson et al., 2001; Newman et. al., 2003], structural dynamics [Wang, and Apte, 2006; Garza and Millwater, 2015], and nonlinear structural analysis [Kim, Ryu, Cho, 2011], among others.

\section{Complex variable finite element method}

The distinguishing characteristic of ZFEM is that complex variable nodal coordinates are used. That is, each node has real and complex values. The real nodal coordinates define the spatial location of the nodes (the same as in traditional finite element methods) and the imaginary nodal coordinates define a "perturbation" of the real spatial nodal coordinates, with the specific perturbation defining the derivative to be computed.

As examples, Figure 1a shows a horizontal perturbation of a hole in a structural component. In this example, the imaginary $\mathrm{X}$ coordinates of all nodes with red arrows are set to the step size value $h$. The mid-side nodes with a blue arrow have imaginary X axis coordinates of $h / 2$. The X coordinates for all nodes along and outside of the green line are zero. The Y coordinate values for all nodes are zero. As mentioned previously, $h$ is typically set at $10^{-10}$ times the smallest element size. Hence this analysis would produce derivatives with respect to the horizontal location of the hole. 
Figure $1 \mathrm{~b}$ shows a radial perturbation of the hole. In this case, the imaginary $\mathrm{X}$ and $\mathrm{Y}$ coordinates of all nodes with red arrows are set in the appropriate ratio such that the resultant is a radial vector of magnitude $h$. The mid-side nodes with a blue arrow have imaginary X and Y coordinate values such that the resultant is a radial vector of magnitude $h / 2$. The imaginary $\mathrm{X}$ and $\mathrm{Y}$ coordinates for all nodes along and outside of the green line are zero. Hence this analysis would produce derivatives with respect to the hole radius. As shown, the user can apply an arbitrary perturbation to the geometry to compute any shape derivative by defining the appropriate numerical values for the imaginary coordinates through the input file. An analogous procedure would be conducted for three-dimensional analyses. This generic shape sensitivity method can be specialized to implement a virtual crack extension by inputting the appropriate imaginary coordinates to extend the crack.

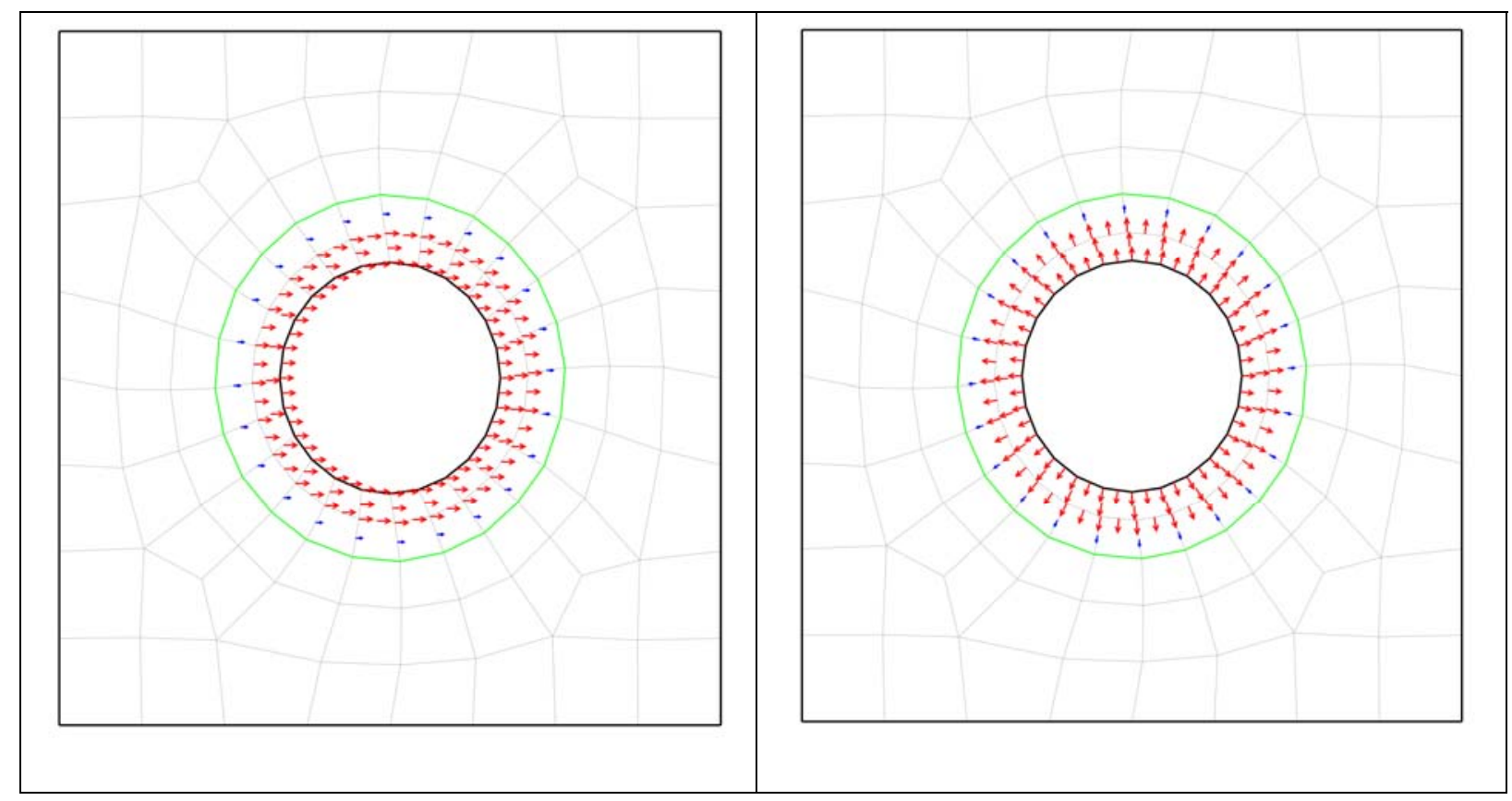

Figure 1: a) Horizontal perturbation of the hole location, b) Radial perturbation of the hole

ZFEM elements use the same shape functions of a standard isoparametric element, e.g.,

$$
x^{*}=\sum_{i}^{n_{\mathrm{Re}}} N_{i}(\xi, \eta) x_{i}^{*}
$$




$$
\begin{gathered}
y^{*}=\sum_{i}^{n_{\mathrm{Re}}} N_{i}(\xi, \eta) y_{i}^{*} \\
z^{*}=\sum_{i}^{n_{\mathrm{Re}}} N_{i}(\xi, \eta) z_{i}^{*}
\end{gathered}
$$

where $x_{i}^{*}=x_{i}+i x_{i}, y_{i}^{*}=y_{i}+i y_{i}, z_{i}^{*}=z_{i}^{*}+i z_{i}$ are the complex nodal coordinates, $N_{i}(\xi, \eta)$ denotes the standard set of shape function, and $n_{\mathrm{Re}}$ is the number of real nodes in the element. Figure 2 shows an example of a 16 noded quadratic quadrilateral element; 8 real (black) and 8 imaginary (yellow) nodes.

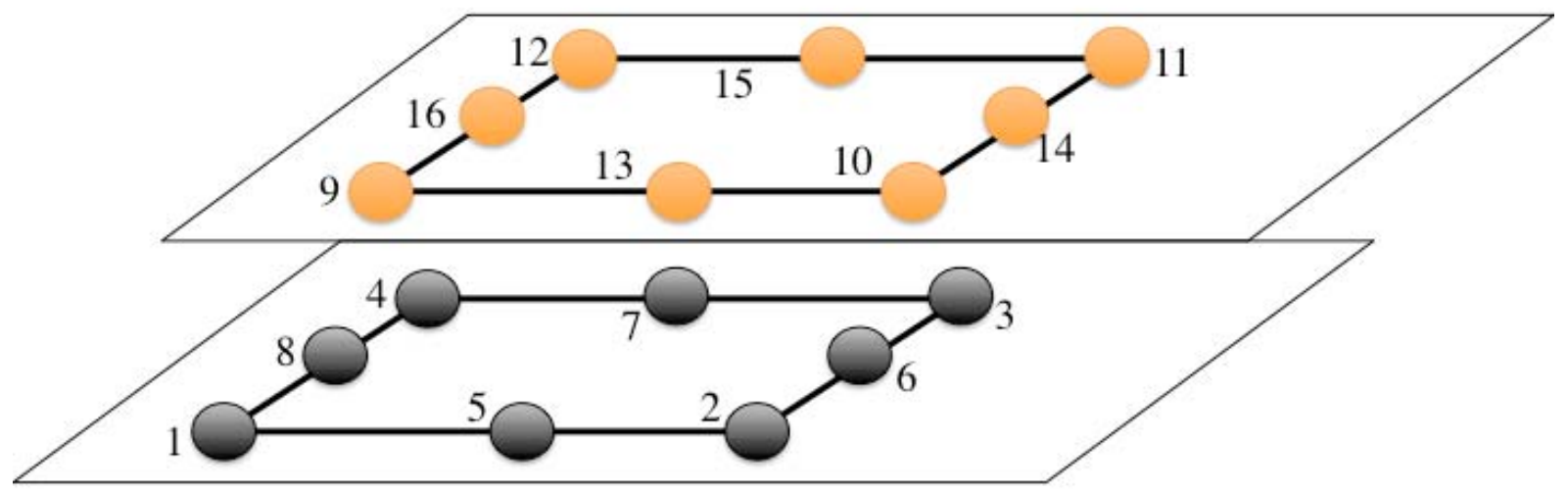

Figure 2 Schematic of a complex variable quadratic 16-noded element with nodes 1-8 real and nodes 9-16 imaginary

The complex displacement vector is of the form $\boldsymbol{u}^{*}=\boldsymbol{u}_{\mathrm{Re}}+\boldsymbol{u}_{\mathrm{Im}} \cdot i$, where $\boldsymbol{u}_{\mathrm{Re}}$ represents the traditional physical displacement and $\boldsymbol{u}_{\mathrm{Im}}$ represents the imaginary displacement. The displacement interpolation in the $\mathrm{X}, \mathrm{Y}, \mathrm{Z}$ directions over complex coordinates $u^{*}, v^{*}, w^{*}$ is defined as:

$$
\begin{gathered}
u^{*}=\sum_{i}^{n_{\mathrm{Re}}} N_{i}(\xi, \eta) u_{i}^{*} \\
v^{*}=\sum_{i}^{n_{\mathrm{Re}}} N_{i}(\xi, \eta) v_{i}^{*}
\end{gathered}
$$




$$
w^{*}=\sum_{i}^{n_{\mathrm{Re}}} N_{i}(\xi, \eta) w_{i}^{*}
$$

Given the complex coordinates, the traditional finite element computations can be carried out, e.g., the strain-displacement matrix and the stiffness matrix all computed according to conventional finite element methods; however, all quantities related to nodal coordinates, including the stiffness matrix, are now complex, e.g.,

$$
\boldsymbol{K}^{*}=\int \boldsymbol{B}^{\mathrm{T}} * \boldsymbol{D} \boldsymbol{B} * \mathrm{~J} * \mathrm{~d} \xi \mathrm{d} \eta
$$

Note, in Eq. (5), the constitutive matrix D is not complex. Sensitivities with respect to material parameters can be obtained by constructing a complex constitutive matrix and perturbing a material property. In that case, all imaginary nodal coordinates would be zero since no geometry is perturbed.

Upon completion of the analysis, the additional degrees-of-freedom available at each node contain the derivative of the displacement at that node with respect to the perturbed parameter times the step size. Hence, the displacement derivative is recovered as shown in Eq. (2). For example, the derivative of the $\mathrm{X}$ displacement at node 1 would be computed as, $\partial \boldsymbol{u}_{1} / \partial \theta \approx \operatorname{Im}\left[\boldsymbol{u}_{1}\right] \cdot h$, where $\boldsymbol{u}_{1}$ is the complex displacement at node 1 in the $\mathrm{X}$ direction, and $h$ is the step size used for the perturbation.

Once the complex displacement field is computed, post-processing can proceed in the traditional manner to obtain strains, $\varepsilon^{*}=\boldsymbol{B}^{*} \boldsymbol{u}^{*}$, stresses, $\boldsymbol{\sigma}^{*}=\boldsymbol{D} \boldsymbol{\varepsilon}^{*}$ and strain energy, $U^{*}=\frac{1}{2} \boldsymbol{u}^{T^{*}} \boldsymbol{K}^{*} \boldsymbol{u}^{*}$, where the superscript T denotes the non-Hermitian (non-conjugate) transpose. The imaginary component of all quantities contains the derivative with respect to the perturbed parameter, e.g., crack area. In particular, the complex strain energy contains the strain energy and its derivative, i.e., the real part of the strain energy variable contains the strain energy estimate for each element and the imaginary component contains the energy release rate times the step size $h$. That is 
$d U / d A=-G=1 / h \operatorname{Im}\left[U^{*}\right]$. The energy release rate for the structure is then obtained by summing the element contributions

$$
G=-\frac{1}{h} \sum_{e l=1}^{n} \operatorname{Im}\left[U^{*}{ }_{e l}\right]
$$

where $\operatorname{Im}\left[U^{*}{ }_{e l}\right]$ denotes the imaginary component of the elemental strain energy, $h$ is the step size used when perturbing the crack, and the summation is carried over all elements.

\section{Boundary conditions}

The boundary conditions specified for the problem must be enforced on the real and imaginary degrees of freedom. For example, if displacement in the $\mathrm{X}$ direction is constrained to be zero at node 1 , both $\operatorname{Re}\left[u_{1 x}\right]=0$ and $\operatorname{Im}\left[u_{1 x}\right]=0$. Other boundary conditions are handled analogously.

Nodal point forces are only applied to the real node; no load is applied to the imaginary node. If nodal forces are applied to an imaginary node, these forces act to perturb the nodal force applied to the real node.

\section{Solving the system of equations}

The global assembly of the stiffness matrix proceeds as in the traditional finite element method. However, solving the system of equations requires a complex solver. Hence a direct complex-valued Gauss-elimination solver can be employed.

Alternatively, since complex variables can be represented in a Cauchy-Reimann matrix formulation, e.g., $a+b i$, can be represented in matrix form as $\left\lfloor\begin{array}{cc}a & -b \\ b & a\end{array}\right\rfloor$, the stiffness matrix can be represented as an all-real element matrix, e.g.,

$$
\boldsymbol{K}=\left[\begin{array}{cc}
\operatorname{Re}\left[\boldsymbol{K}^{*}\right] & -\operatorname{Im}\left[\boldsymbol{K}^{*}\right] \\
\operatorname{Im}\left[\boldsymbol{K}^{*}\right] & \operatorname{Re}\left[\boldsymbol{K}^{*}\right]
\end{array}\right]
$$

and solved using a real-valued unsymmetric solver. 


\section{Perturbation of the Crack Length or Crack Area}

In ZFEM, derivatives with respect to the crack length (2D) or area (3D) can be obtained by using the imaginary nodal coordinates. As an example, Figure 3 shows a 2D fracture problem with the crack (red line) lying along the $\mathrm{X}$ axis with symmetry used along the $\mathrm{Y}=0$ axis. A self-similar virtual crack extension is implemented by elongating the crack a small amount using the imaginary $\mathrm{X}$-axis coordinates. A procedure to do so is as follows. The green line marks the boundary between the perturbed and unperturbed regions; the X-axis imaginary coordinates along the green line and all nodes outside of the green line are set to 0 . The $\mathrm{X}$-axis imaginary coordinates for the dashed transition nodes (typically mid-side nodes) are moved by $1 / 2 h$. The inner white region is moved as a rigid body by setting the $\mathrm{X}$-axis imaginary coordinates for all nodes to a value of $h$ (typically $10^{-10}$ times the size of the smallest element). The Y-axis coordinate for all imaginary nodes is set to zero.

Figure 4 shows a three-dimensional view of the $2 \mathrm{D}$ perturbed region. The amount of the imaginary $\mathrm{X}$-axis perturbation is plotted along the third axis of the mesh. It is evident that the perturbation is analogous to the "q" function employed in the J integral calculations [Anderson, 1991]. Note, however, that in the domain-based $\mathrm{J}$ integral formulation, only elements for which $d q / d x \neq 0$ contribute to the energy release rate, whereas for ZFEM, all elements contribute. That is, $d q / d x$ has no meaning for ZFEM.

For 3D cracks, the entire crack front can be perturbed for an average energy release rate or local perturbations can be implemented. Figure 5 shows an example of the perturbation of a 3D crack front where a region of nodes (red color) is perturbed radially. Analogously to 2D perturbations, a region of nodes is perturbed by $h$, a layer of transition nodes is perturbed $1 / 2 h$ and all other nodes are left unperturbed. Figure 6 shows the local perturbation along the crack front using a triangular perturbation.

Alternate "q" functions have been studied such as a smoothly varying pyramid function rather than the plateau-like function in Figure 4, but provide no advantage. Hence the plateau-like function is used for computations. In general, the same $q$-function that represents a virtual 
displacement of a finite element mesh for a domain J-integral calculation may be used to apply a small imaginary displacement for ZFEM.

In our implementation, all coordinates, real and imaginary, are read from an input file. Hence the desired VCE is implemented through a preprocessing step that selects the node set of the perturbed region; typically using a graphical preprocessor.

The size of the perturbation domain has been studied empirically. In general, the ZFEM results are largely independent of the perturbed domain. That is, different perturbation regions can be used with similar (mostly identical) resulting energy release rate values. Numerical examples indicate that the energy release rate results are slightly more accurate the larger the perturbed region; however the effect is minor. In some cases, even perturbing only the crack tip gives good results; but this is not recommended. It is recommended that a minimum of a few layers of elements around the crack tip, say 4 or 5, be perturbed; however, more elements can be perturbed with good results. In addition, it is not necessary to use a rosette of elements around the crack tip; although good modeling of the crack tip, e.g., quarter-point elements, is recommended.
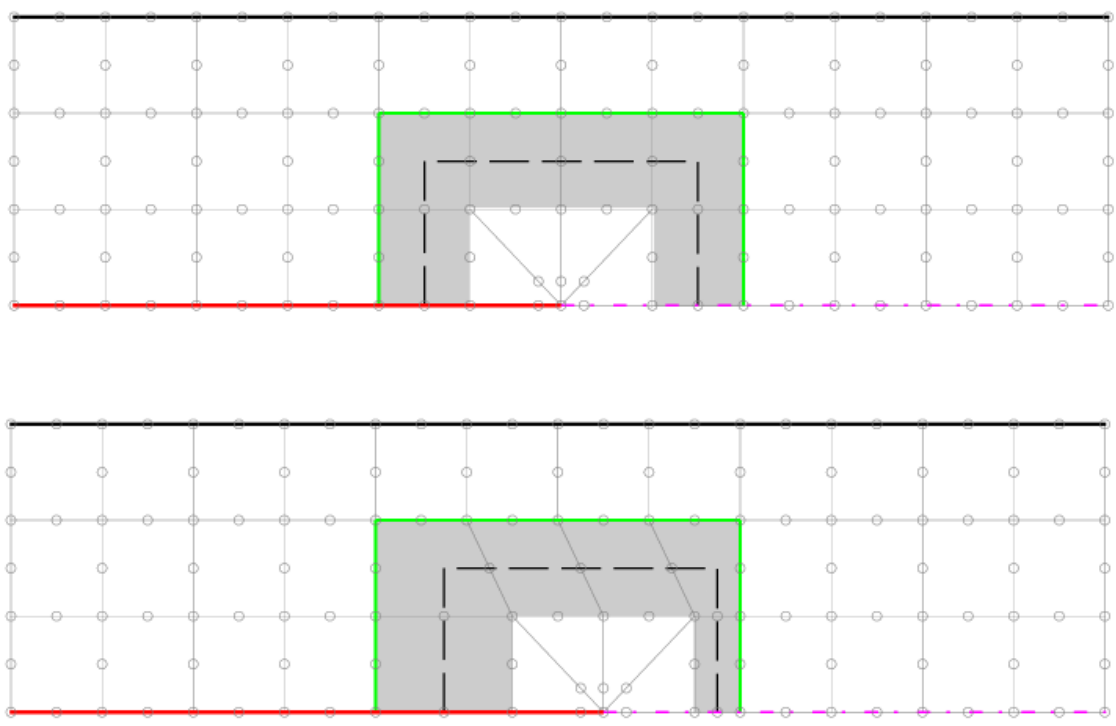

Figure 3 Schematic of crack extension 


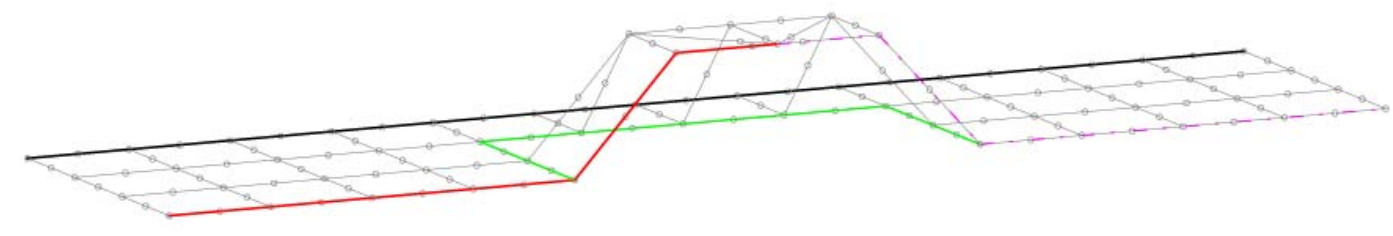

Figure 4 Schematic of " $q$ " function defining the virtual crack extension

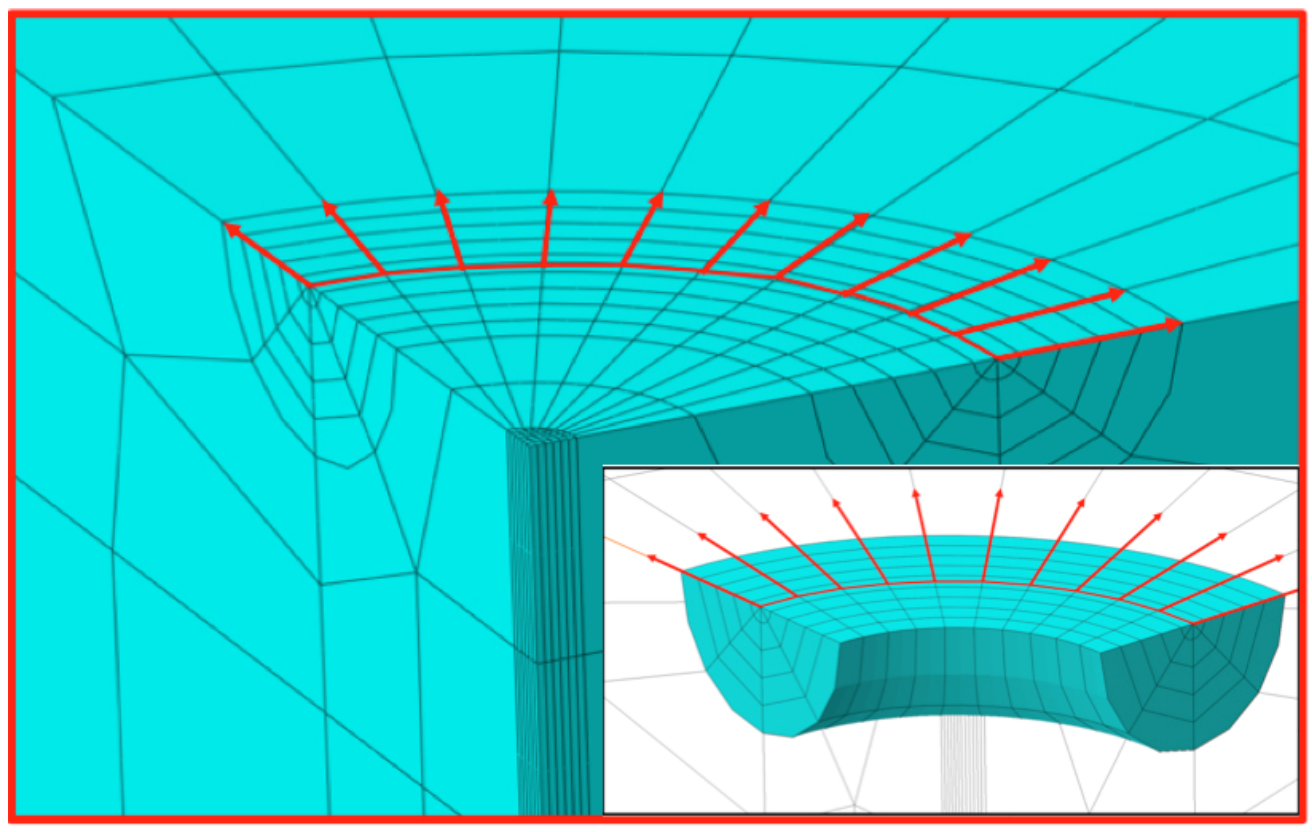

Figure 5 Schematic of $3 D$ crack front perturbation 


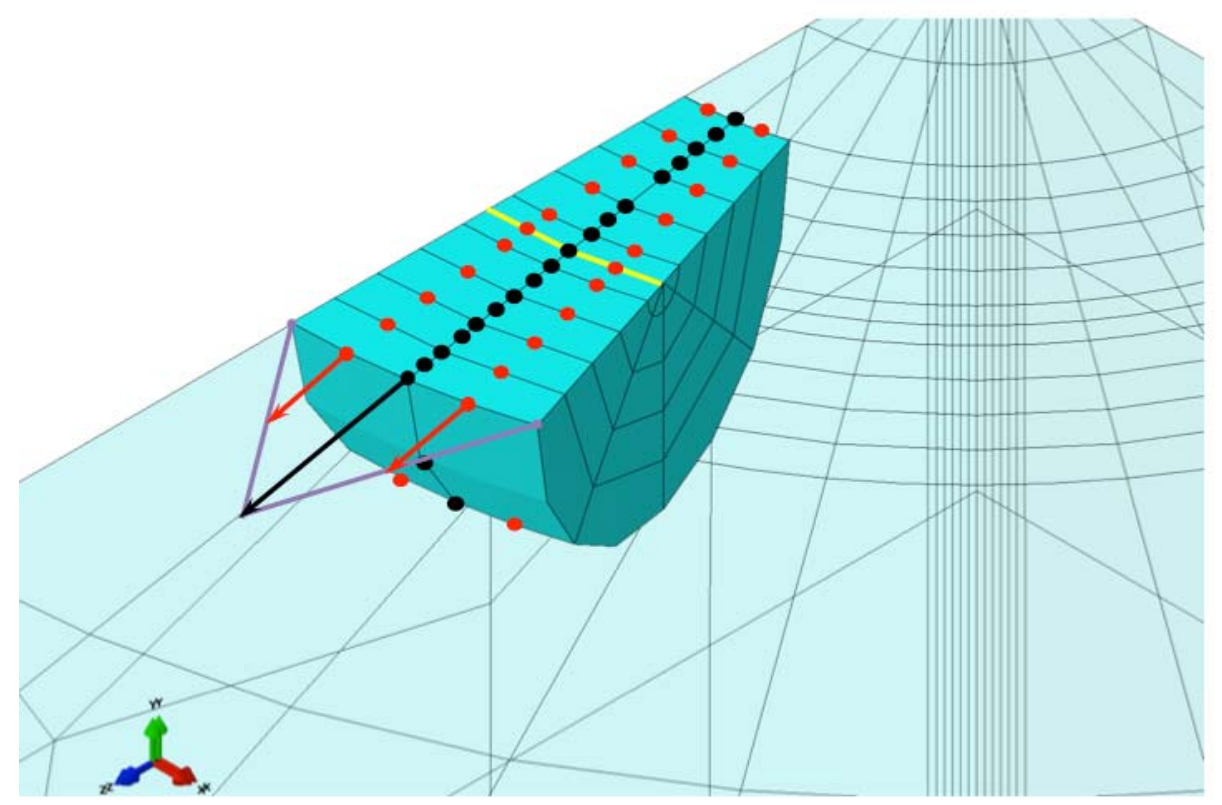

Figure 6 Local perturbation along the 3D crack front

\section{Numerical Examples}

2D plane strain, axisymmetric, and 3D numerical examples are presented. All problems were solved with 16 noded (8 real, 8 imaginary) quadratic quadrilateral elements. Quarter point elements were used at the crack tip [Tracey, 1971]. ZFEM does not require a "spider-web" mesh design around the crack tip but that design was used for some problems, in particular to also compute the $\mathrm{J}$ integral for comparison purposes. For all example problems, ZFEM was implemented as a User Element (UEL) within the Abaqus commercial finite element program [Abaqus, 2015].

\section{Center-cracked panel under crack face loading}

The energy release of a crack in an isotropic center-cracked panel under crack face loading was modeled using ZFEM. Using symmetry, one quarter of the model was meshed. The plane strain finite element model consisted of 459116 noded ( 8 real, 8 imaginary) quadratic quadrilateral elements and 14184 nodes. Quarter point elements were used at the crack tip. The model was 
loaded under crack face pressure loading of $\sigma=0.68948 \mathrm{MPa}(100 \mathrm{psi})$. The finite element mesh in the region of the crack is shown in Figure 7.

Figure 8 shows a close up of the crack tip finite element mesh. The perturbed region is highlighted in green. As discussed previously in the section describing the crack perturbation method, nodes on and outside the green line were unperturbed; mid-side nodes of the adjacent elements were perturbed by $h / 2$; all other nodes inside the green line were perturbed by a step size $h$ in the $\mathrm{X}$ direction. The Y-axes imaginary coordinates of all nodes was set to zero. The numerical values used in the analysis were: crack length to width ratio $(a / W=0.3)$, overall model height $(H=$ 6), elastic modulus $\left(E=6.8948 \mathrm{E} 5 \mathrm{GPa}\left(100 \times 10^{9} \mathrm{psi}\right)\right.$, Poisson's ratio $(v=0.3)$, and perturbation step size $h=10^{-15}$.

Unlike other formulations, ZFEM requires no additions to the fundamental equations for problems with crack faced loading. However, if pressure is applied to the crack faces, then the increase in nodal load due to the increase in crack area caused by the crack extension should be considered to obtain good accuracy. This load increase can be implemented as a perturbation by applying the appropriate nodal loading values to the imaginary nodes that comprise the crack face. For this problem, the increase in nodal loads due to the extension of the crack is $\Delta P=\sigma \cdot h$. This increase of loads was applied to the imaginary nodes along the crack face lying within the perturbed region in a manner per the quadratic element formulation, i.e., the loads were computed and applied as consistent nodal loads.

The secant formula was also used to calculate the stress intensity factor $\left(K_{R e f}\right)$ for comparison purposes [Anderson, 1991]

$$
K_{\mathrm{Ref}}=\sigma \sqrt{\pi a} \sqrt{\sec \left(\frac{\pi \mathrm{a}}{2 \mathrm{~W}}\right)}\left[1-0.025\left(\frac{\mathrm{a}}{\mathrm{W}}\right)^{2}+0.06\left(\frac{\mathrm{a}}{\mathrm{W}}\right)^{4}\right] .
$$

Table 2 shows the comparison of the K solutions between: the reference $\mathrm{K}$ solution, (KRef), the ZFEM model under crack face pressure, the ZFEM model under remote tension, and the $\mathrm{K}$ solution developed from the Abaqus J integral result under crack face pressure. As clearly shown, 
the ZFEM result agrees the other solutions. The q function chosen for the Abaqus J integral calculation was the same as used for the ZFEM perturbation.

\begin{tabular}{|l|c|c|}
\hline \multirow{2}{*}{ Analysis Method } & $\begin{array}{c}\text { Stress Intensity } \\
\text { Factor }\end{array}$ & $\begin{array}{c}\text { Discrepancy from } \\
\text { Secant Formula }\end{array}$ \\
\cline { 2 - 3 } Reference Secant Formula $\left(\boldsymbol{K}_{\text {Ref }}\right)$ & $\boldsymbol{K} / \boldsymbol{\sigma}$ & $\left(\boldsymbol{K}-\boldsymbol{K}_{\text {Ref }}\right) / \boldsymbol{K}_{\text {Ref }}$ \\
\hline ZFEM Remote Tension & 1.026664038 & - \\
\hline ZFEM Crack Face Pressure & 1.026877369 & $2.08 \times 10^{-4}$ \\
\hline Abaqus Crack Face Pressure & 1.026877368 & $2.08 \times 10^{-4}$ \\
\hline
\end{tabular}

Table 1 Results from CCP analysis

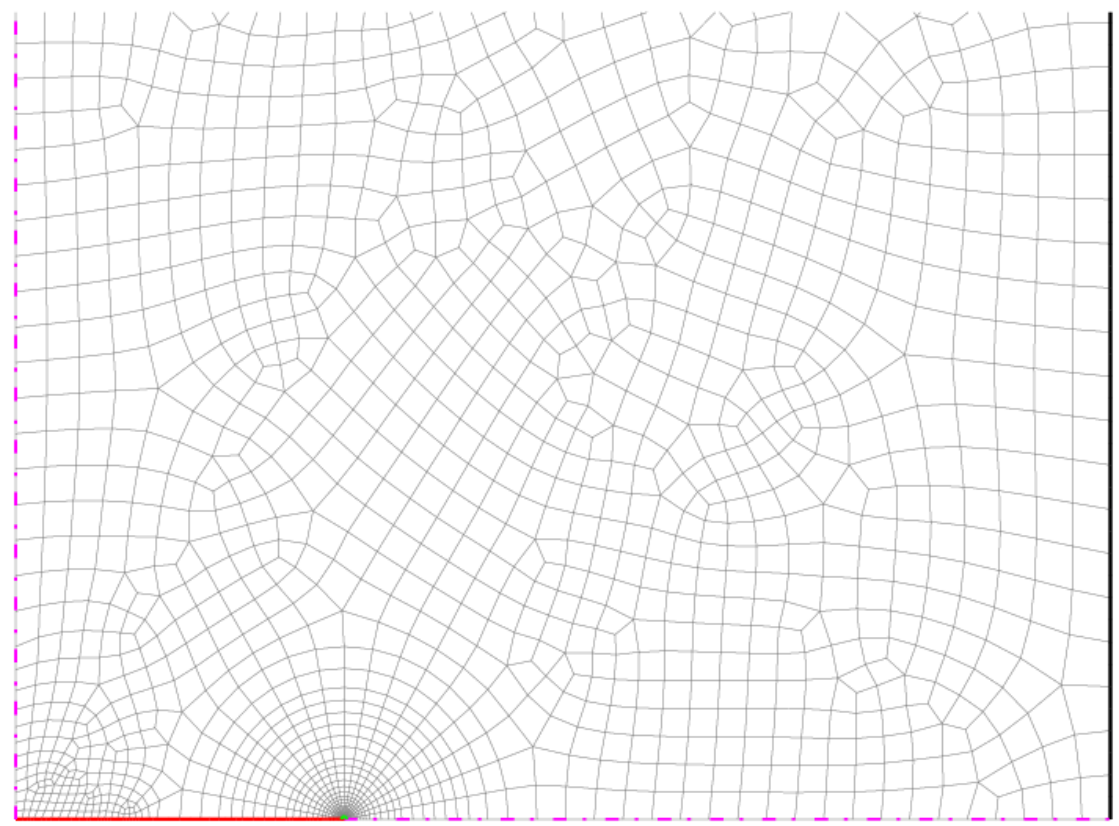

Figure 7 Finite element mesh details of center-cracked panel example 


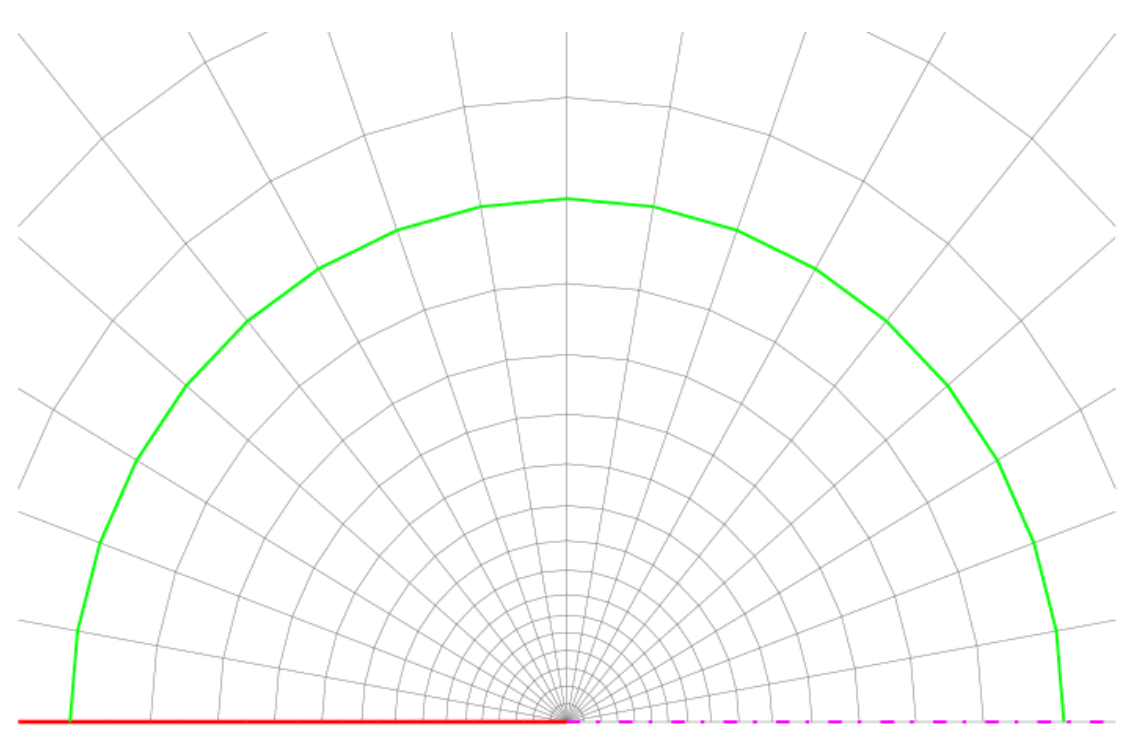

Figure 8 Close up of crack tip finite element mesh of center-cracked panel example

\section{Double-cantilever Beam, Convergence Study}

A symmetric isotropic plane strain double-cantilever beam (DCB) model was defined by its rectangular section height $(H)$, overall model width $(W)$, arm length $(a)$, and the elastic modulus $(E)$. A two-dimensional plane stress finite element model was created using symmetry plane along the ligament, see Figure 9. A symmetric end-moment $(M)$ was applied as shown.

The analytical strain energy release rate for a linear-elastic isotropic double-cantilever beam under symmetric end-moment loading can be derived from beam theory and an elastic foundation model and is given below as

$$
G_{\text {Analytical }} \equiv-\frac{\partial U}{\partial a}=\frac{12 M^{2}}{E H^{3} T^{2}}
$$

The discretization of the finite element model was varied to observe the convergence of the strain energy release rate computations compared to the theoretical reference value. A series of analyses was conducted by successively increasing the number of elements across the dimension $\mathrm{H}$, with $\mathrm{H}$ and all dimensions unaltered, and keeping square elements throughout the model. The beam slenderness ratio $a / H=5$ was used with an overall model aspect ratio of $W / H=10.16$ noded ( 8 real, 8 imaginary) quadratic quadrilateral elements were used with quarter-point elements at the crack tip; however a near-tip element rosette was not used. Numerical values used were $M=1 \mathrm{E} 6, E=100 \mathrm{E} 9, T$ 
$=1$. Figure 10 shows the finite element mesh modeled with 3 elements across $\mathrm{H}$. The perturbation used for all the virtual crack extension cases was $H$ horizontally and $2 H$ vertically, see the green line in Figure 10.

Figure 11 shows the relative error in $G$ between the ZFEM and analytical solutions, i.e., Error $=\frac{G_{\text {Zfem }}-G_{\text {Analytical }}}{G_{\text {Analytical }}}$ as a function of the number of elements across H. The calculation for $G$ improves with each mesh refinement. That is, the CTSE method does not limit the accuracy obtainable when computing the energy release, and the discretization of the finite element mesh controls the accuracy. This is consistent with other publications showing convergence of the stress derivatives with respect to mesh discretization [Voorhees, et al., 2011].

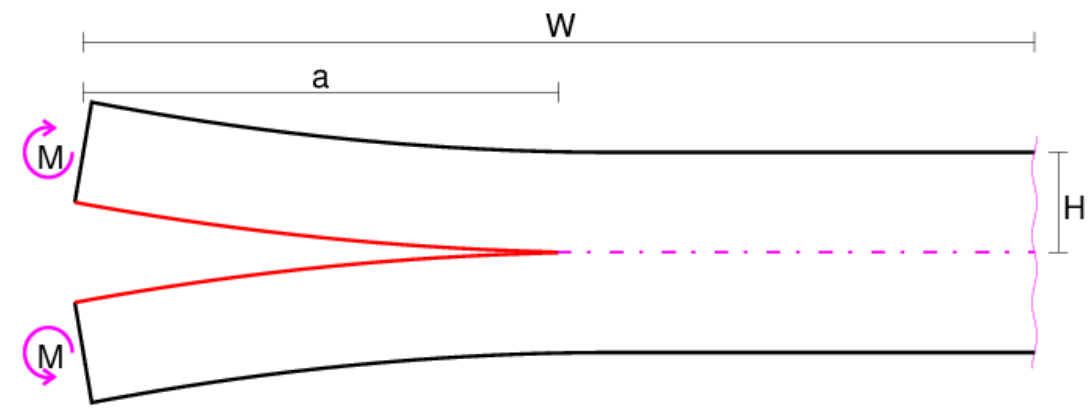

Figure 9 Schematic of DCB Example

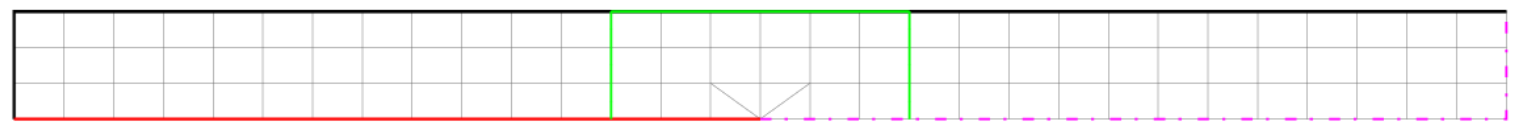

Figure 10 Finite Element mesh for DCB example with 3 elements across $H$ (perturbed region boundary in green) 


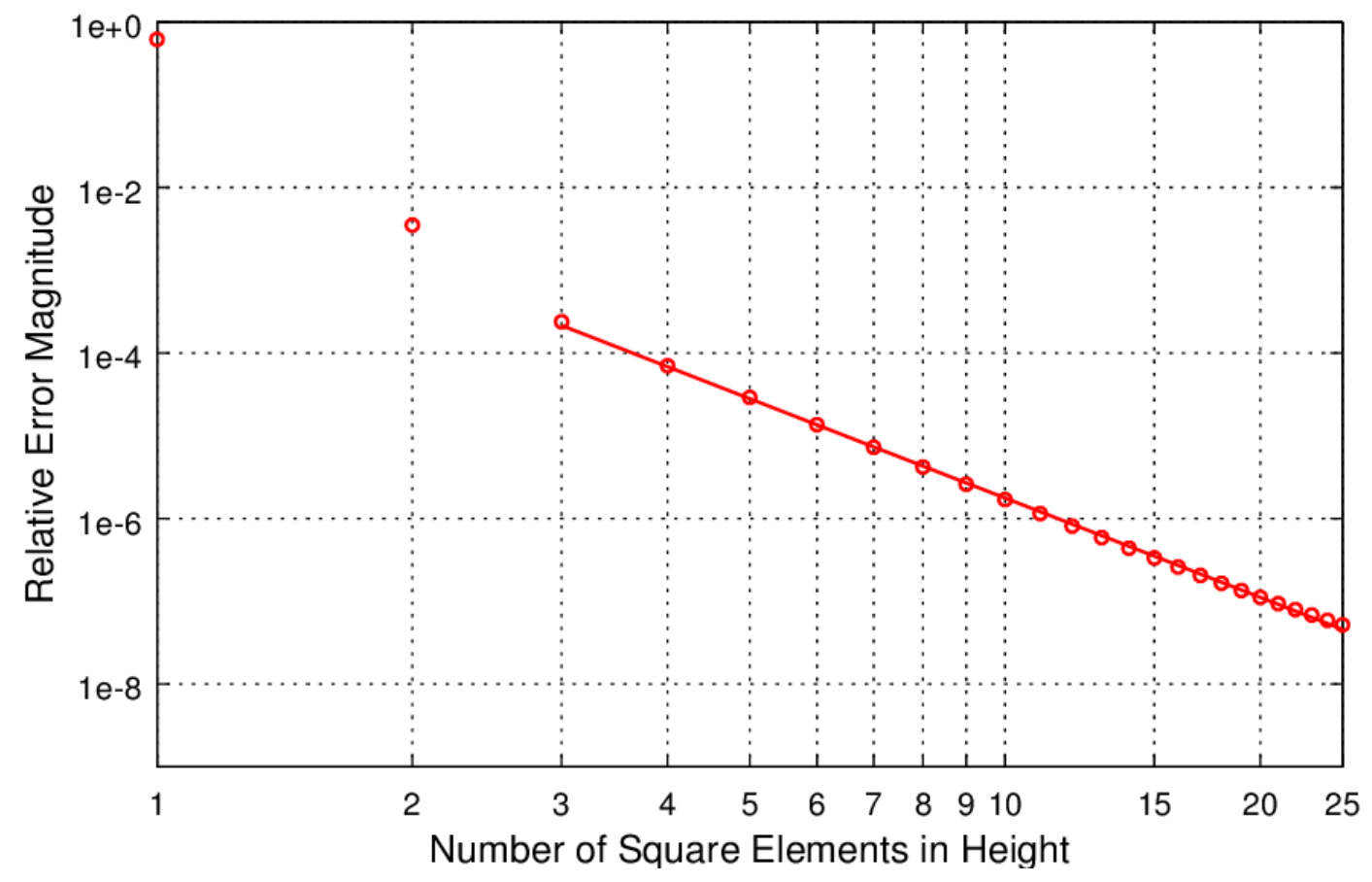

Figure 11 Relative error in $G$ vs analytical result

\section{Axisymmetric penny shaped crack}

The energy release rate for a penny shaped crack was computed using an axisymmetric 16 noded ( 8 real, 8 imaginary) quadratic quadrilateral element. A schematic of the problem is shown in Figure 12. The finite element model with the near tip mesh are shown in Figures 13 and 14, with the green line showing the extent of the perturbation region.

The geometry consisted of a bar with diameter of $101.6 \mathrm{~cm}$ [40 in] and a height of $152.4 \mathrm{~cm}$ [60 in]. The dimension of the penny shaped crack was 50.8 [20 in] $(\mathrm{a} / \mathrm{D}=1 / 2)$. The bar was loaded under a uniform tension of $689.5 \mathrm{KPa}[100 \mathrm{psi}]$ applied to the top surface. Symmetry about $\mathrm{z}=0$ was used such that only the top half of the bar was modeled. The finite element model contained 1939 nodes and 618 elements. The perturbation region boundary is shown as a green line; nodes on the green boundary are not perturbed; all nodes one element inwards from the green boundary are perturbed by $h$; the midside node near the green boundary is perturbed $1 / 2 h$. For this problem, the perturbation step size used $6.5 E-12$ (1E-10 times the element size at the crack tip). 
As the model revolves around the z-axis, the resulting change in area due to the perturbation was an annular ring shown in Figure 11 and defined as $\Delta \mathrm{A}=\pi\left((\mathrm{a}+\mathrm{h})^{2}-\mathrm{a}^{2}\right)$. The energy release rate is computed as the imaginary portion of the strain energy divided by the change in area $\Delta A$, i.e.,

$$
\mathrm{G}=-\frac{1}{\Delta \mathrm{A}} \sum_{\mathrm{el}=1}^{\mathrm{n}} \operatorname{Im}\left[\mathrm{U}^{*}{ }_{\mathrm{el}}\right]
$$

Table 2 shows the results obtained from ZFEM and $\mathrm{J}$ integral results computed by Abaqus for the first five contours. The ZFEM result is in in excellent agreement with the Abaqus $\mathrm{J}$ integral. Tada et al. provide an approximate solution: $\mathrm{G}=0.7635 \mathrm{~N} / \mathrm{m}(0.00436 \mathrm{lb} / \mathrm{in})$ [Tada, et al., 1973 ].

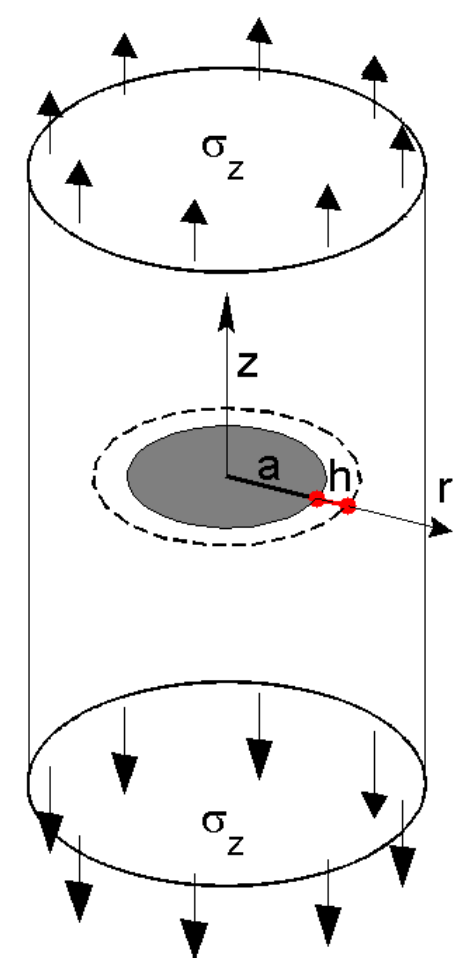

Figure 12 Axisymetric model for the Penny Shaped crack in a round bar and resulting area change due to the applied perturbation 


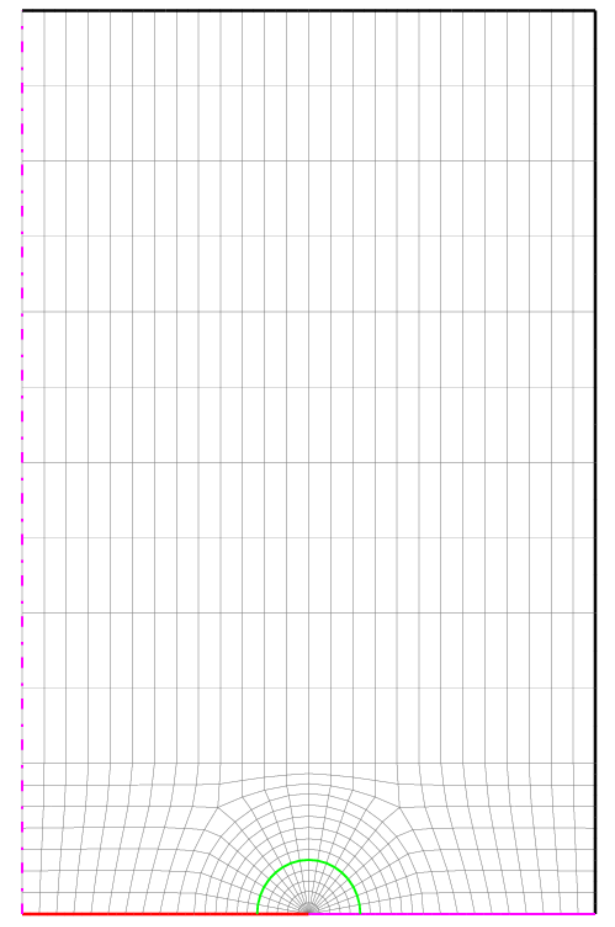

Figure 13 Finite element mesh for the axisymmetric penny shaped crack example

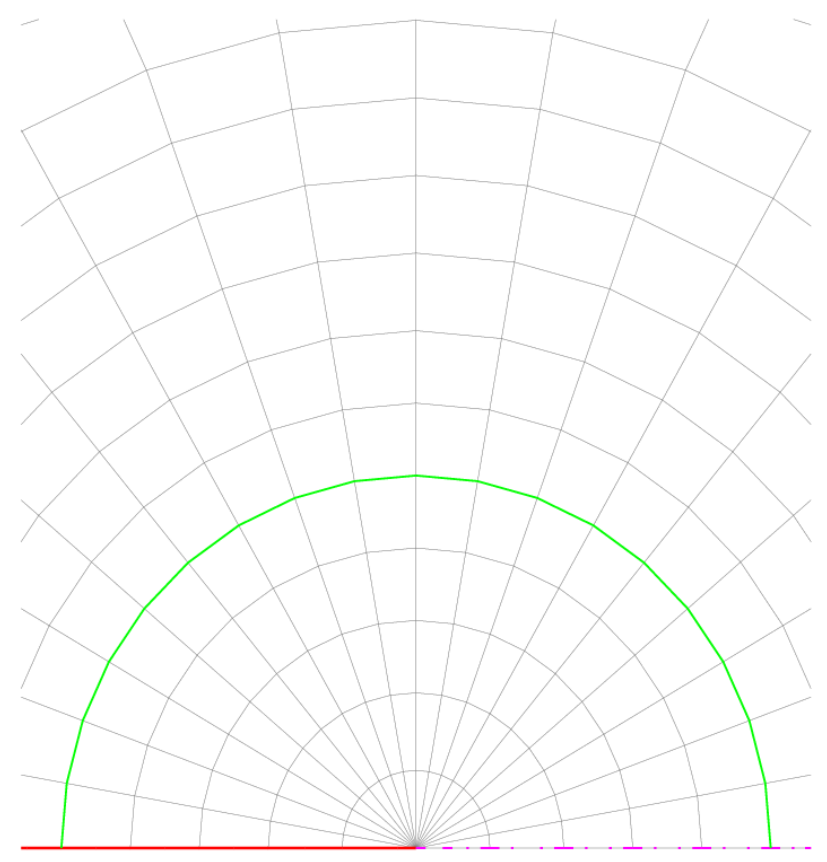

Figure 14 Near tip finite element mesh for axisymmetric penny shaped crack example

\begin{tabular}{|c|c|c|c|}
\hline Contour & Abaqus (J-Integral) & ZFEM & ZFEM/Abaqus \\
\hline $\mathbf{1}$ & 0.786941189 & 0.786556282 & 0.999510882 \\
\hline $\mathbf{2}$ & 0.786231926 & --- & 1.000412545 \\
\hline $\mathbf{3}$ & 0.786554159 & ---- & 1.000002699 \\
\hline $\mathbf{4}$ & 0.786582179 & ---- & 0.999967076 \\
\hline $\mathbf{5}$ & 0.786589184 & ---- & 0.999958171 \\
\hline
\end{tabular}


Table 2 Energy release rate results for the axisymmetric model of a penny shaped crack in a bar.

\section{D Orthotropic Double Cantilever Beam}

A three-dimensional fracture analysis was conducted using an orthotropic DCB specimen under mode I loading conditions with the following dimensions and material properties: length $(W)$ $=150 \mathrm{~mm}$, crack length $(a)=30.5 \mathrm{~mm}$, height $(H)=1.5 \mathrm{~mm}$, width $(T)=25 \mathrm{~mm}$, that is $a / H=4.92$, $W / H=100$. The following material properties of T300/1076 Unidirectional graphite/Epoxy prepreg: $\mathrm{E}_{11}=139.4 \mathrm{GPa}, \mathrm{E}_{22}=10.16 \mathrm{GPa}, \mathrm{E}_{33}=10.16 \mathrm{GPa}, \mathrm{G}_{12}=4.6 \mathrm{GPa}, \mathrm{G}_{13}=4.6 \mathrm{GPa}, \mathrm{G}_{23}=3.54 \mathrm{GPa}, v_{12}$ $=0.3, v_{13}=0.3, v_{23}=0.436$.

The analytical solution for the energy release rate for this problem is [Szekrenyes and $\mathrm{Uj}$, 2006]

$$
G_{I}=\frac{12 P^{2} a^{2}}{b^{2} h^{3} E_{11}}\left[1+0.55\left(\frac{h}{a}\right)\left(\frac{E_{11}}{E_{22}}\right)^{0.25}+0.31\left(\frac{h}{a}\right)^{2}\left(\frac{E_{11}}{E_{22}}\right)^{0.5}+0.32\left(\frac{h}{a}\right)\left(\frac{E_{11}}{G_{12}}\right)^{0.5}+0.1\left(\frac{h}{a}\right)^{2}\left(\frac{E_{11}}{G_{12}}\right)\right]
$$

The mesh contained a total of 125,423 real and 125,423 imaginary nodes modeled with 25,280 40 noded (20 real and 20 imaginary) quadratic elements. The front and back surfaces were constrained in order to mimic plane strain conditions for comparison with the analytical solution.

The crack extension along the $\mathrm{X}$ direction was assumed uniform throughout the width dimension $T$. That is, all of the nodes along the crack front were perturbed uniformly in the $\mathrm{X}$ direction, resulting in the perturbation of 6,154 nodes along the crack front. Figure 15 shows the finite element mesh and a side view along the $\mathrm{Z}$ direction of the crack front. The nodes that were perturbed by the step size $h$ are highlighted in red. Not shown in the figure are the mid-side nodes surrounding the outer ring that were perturbed $h / 2$. The virtual crack area extension was equal to $\Delta A=h \cdot T$. 


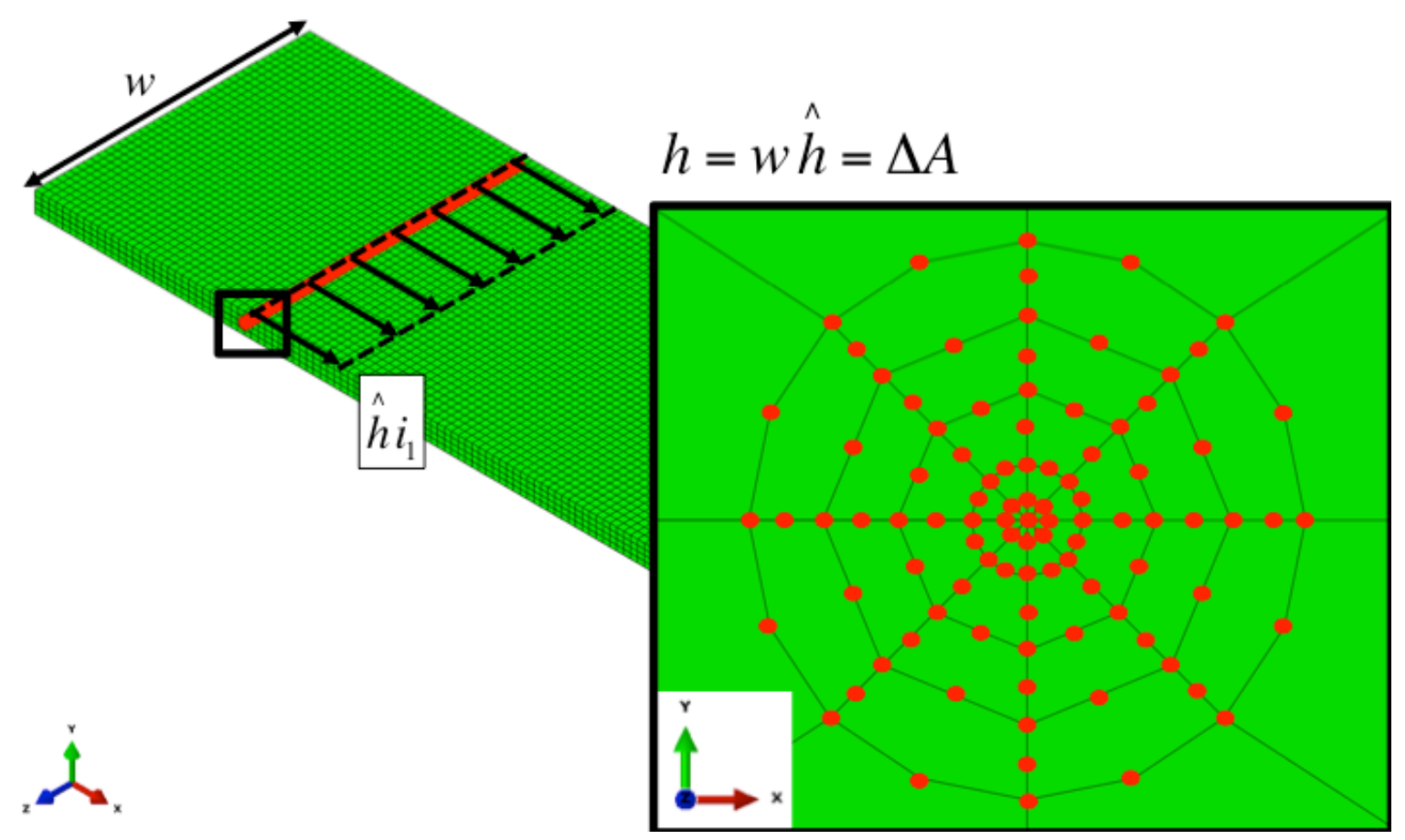

Figure 15 Perturbed nodes for orthotropic DCB example

After performing the analysis using ZFEM, the results were compared to the J-integral results obtained from Abaqus and the analytical solution for an orthotropic DCB. These results are shown in Figure 16. From these results it can be observed that the ZFEM, J integral, and analytical results are in good agreement. 


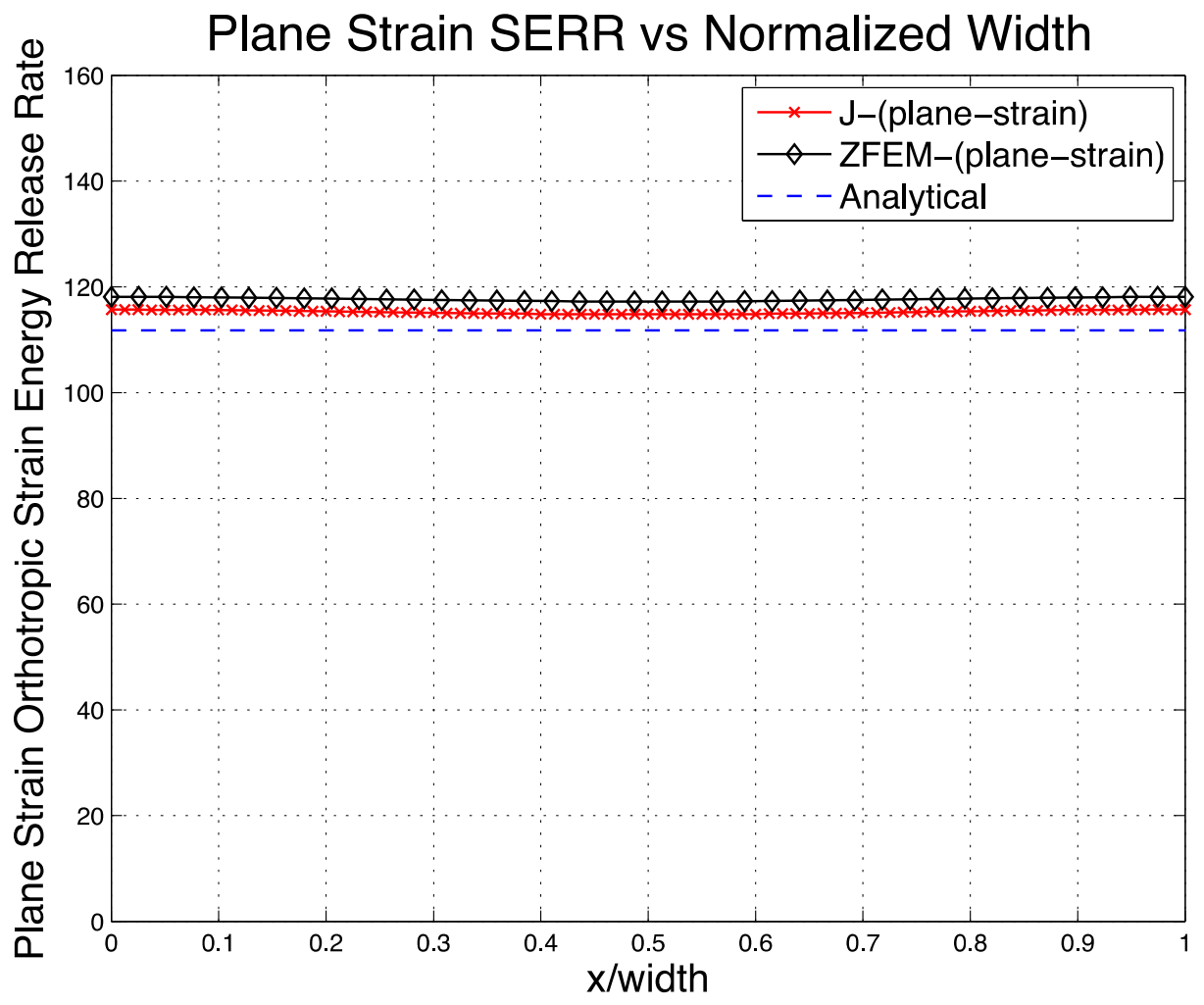

Figure $163 D$ orthotropic DCB stain energy release rate results

\section{D Penny shaped crack in infinite body}

A finite element simulation of an embedded penny shaped crack was solved using 3D 40 noded (20 real, 20 imaginary) quadratic elements using a model of a cylinder under remote tension. The following dimensions and properties were used for the model shown in Figure 12: Radius $(\mathrm{R})=$ $20 \mathrm{~m}$, crack radius $(\mathrm{a})=1 \mathrm{~m}$, cylinder height $(\mathrm{h})=20 \mathrm{~m}$, applied stress $(\sigma)=30 \mathrm{kPa}, \mathrm{E}=104 \mathrm{GPa}, v$ $=0.3$.

Three planes of symmetry were used such that only one-eighth of the full model was meshed, see Figure 17. 3D 40 noded (20 real, 20 imaginary) quadratic isoparametric elements were used. A swept mesh that contained quarter point nodes was placed around the crack front to capture the stress singularity. The mesh used for this example contained a total of 24,480 nodes and 5,365 elements. Boundary conditions were imposed on the left and right wall to constrain translation in 
the $\mathrm{z}$ and $\mathrm{x}$ direction, respectively. The top face, excluding the crack, was fixed to prevent translation in the y direction, and a tension pressure load was applied to the bottom surface.

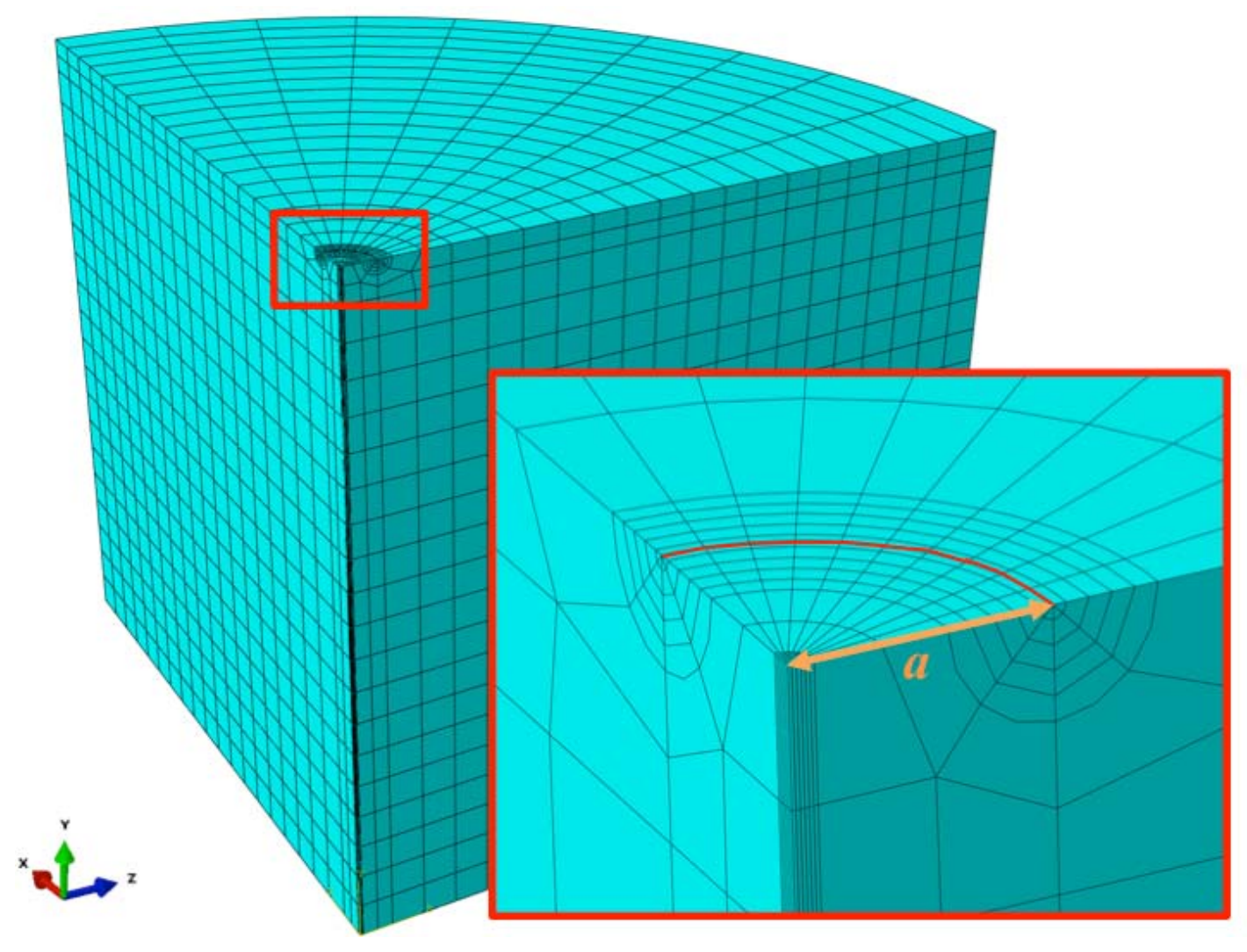

Figure 17 Embedded penny shaped crack finite element model

To compute the energy release rate all of the nodes within the five swept contour regions around the crack front were uniformly perturbed in the radial direction. The nodes that were perturbed and the direction in which the nodes were perturbed are shown in Figure 18. The red nodes were perturbed by a magnitude of $1 \mathrm{E}-10$. The mid-side nodes that surround the boundary of the red nodes, not shown, were perturbed by a magnitude of 5E-11. The red vectors that are normal to the crack front represent the direction in which nodes were perturbed. 


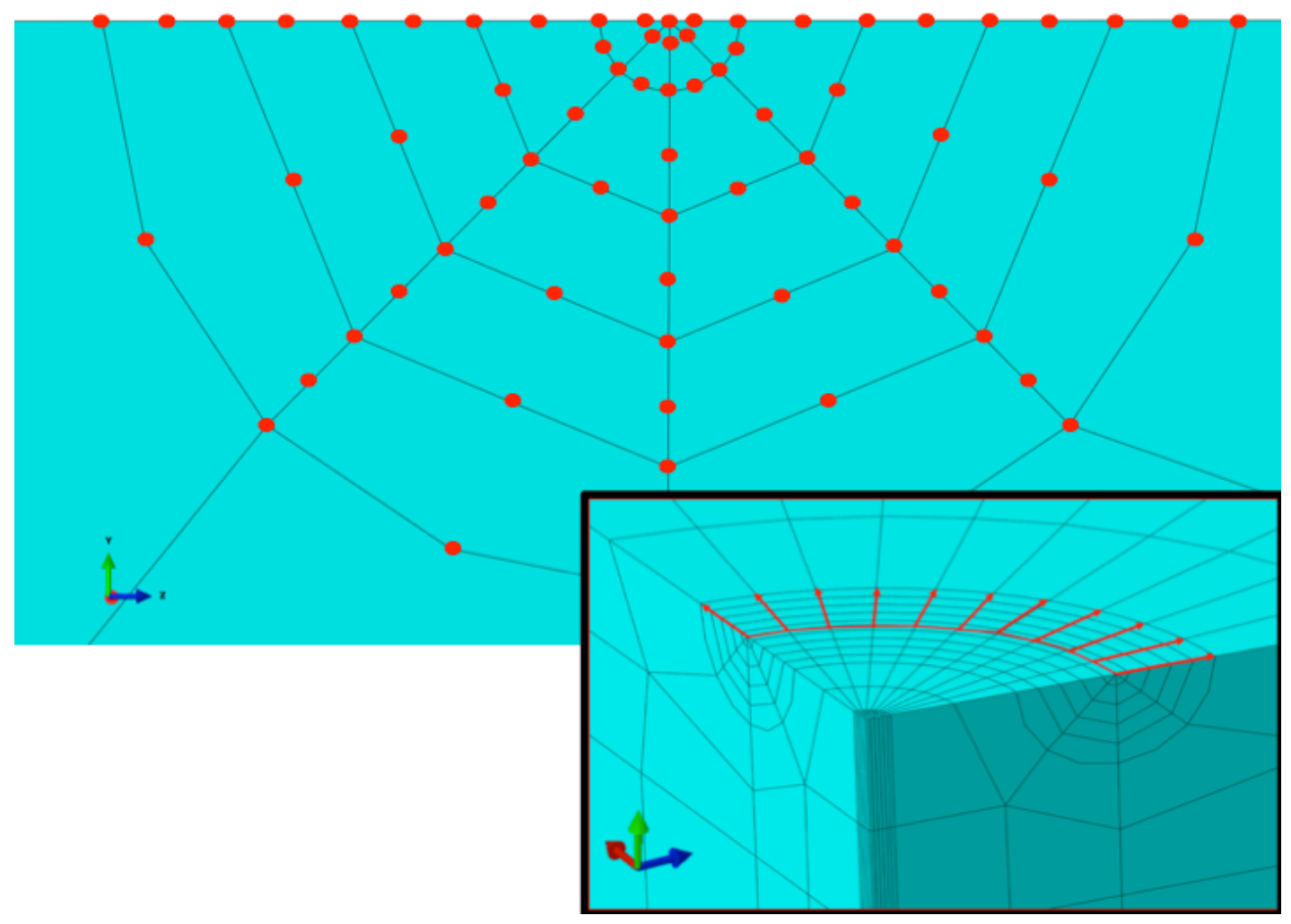

Figure 18 Uniform radial perturbation of the crack front

The analytical solution for a crack in an infinite body is [Sanford, 2003].

$$
K_{I}=2 \sigma \sqrt{a / \pi}
$$

This example was solved using ZFEM and the J-integral capability of Abaqus. The strain energy release rate of both numerical methods was converted into the stress intensity factor. The results for $\mathrm{K}_{\mathrm{I}}$ along the crack front are shown in Figure 19. The figure shows that the ZFEM and J-integral solutions agree closely with the analytical solution. 


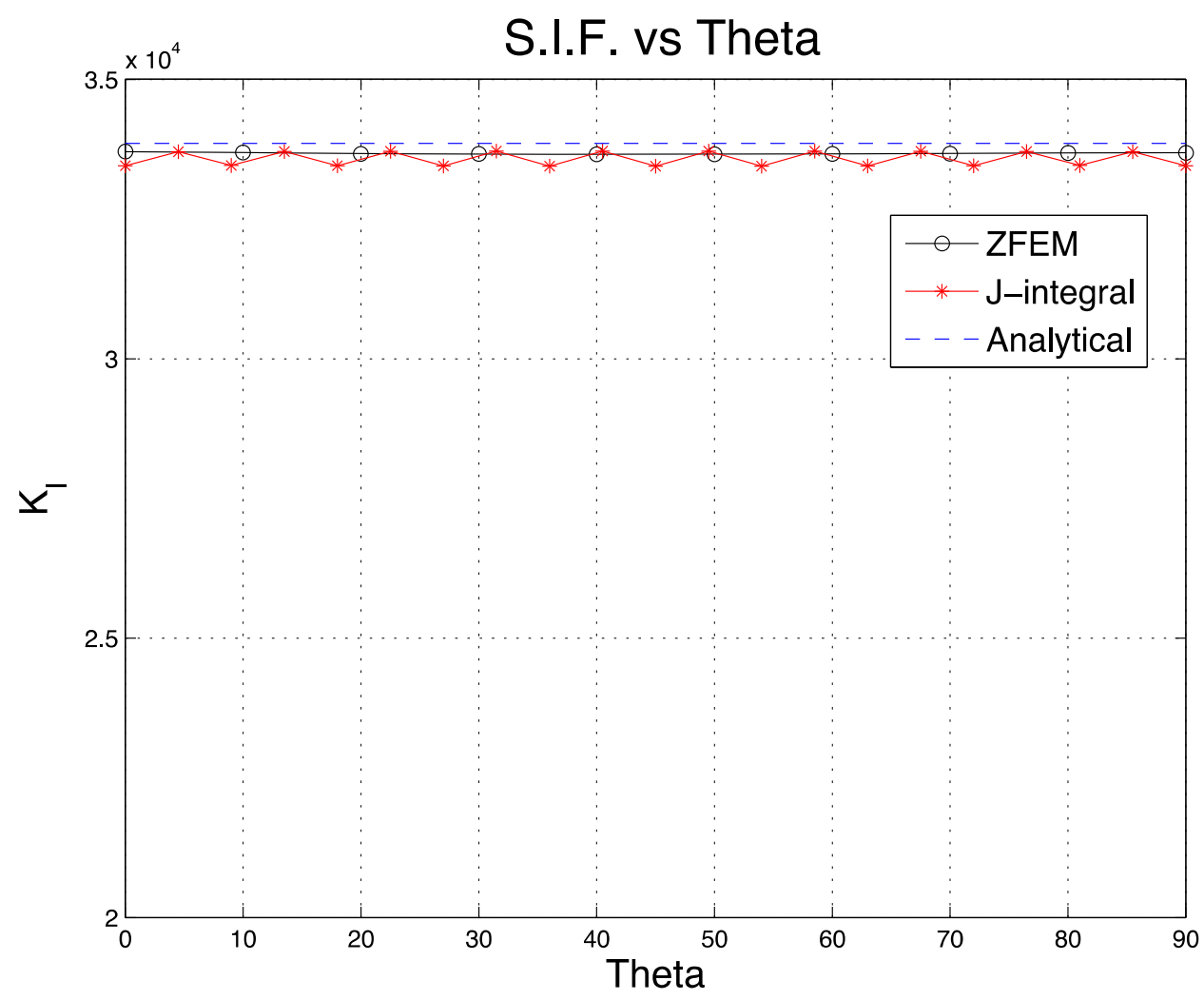

Figure 19 Stress intensity factor along the crack front for embedded penny shaped crack

A second analysis was conducted to compute local energy release rate values along the crack front using a local perturbation. Three different perturbation shapes were considered: i) a triangular perturbation, b) a trapezoidal perturbation, and c) a rectangular perturbation.

Figure 20 shows the nodal perturbations that were applied to the nodes along the crack front to generate a triangular perturbed crack extension. The yellow line denotes the crack front, the red nodes represent the nodes that were perturbed by a step size of $h=1 \mathrm{E}-10$, and the blue nodes are the mid-side nodes that were perturbed by a distance of $1 / 2 h=5 \mathrm{E}-11$. The area of the virtual crack extension was equal to $\Delta A=1 / 2 h *$ (element widths). 


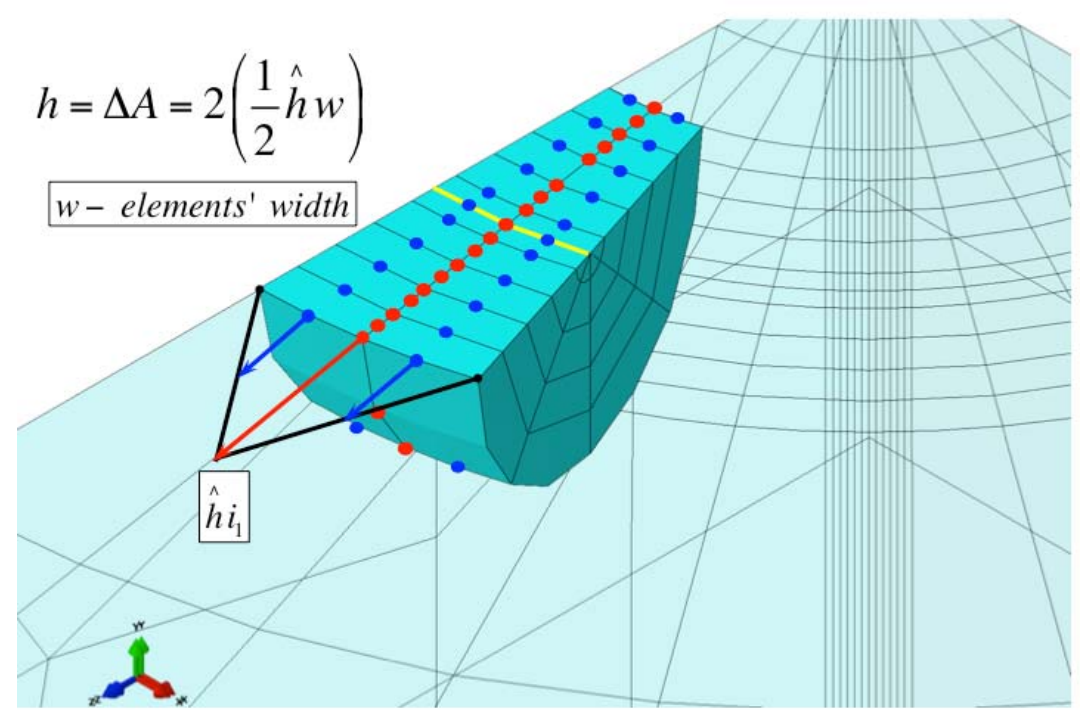

Figure 20 Triangular localized perturbation

The second localized perturbation case the crack was a trapezoidal region, see Figure 21. The nodes highlighted in red were perturbed by a magnitude of $h=1 \mathrm{E}-10$. No other nodes were perturbed. The area of the virtual crack extension was equal to $\Delta A=1 / 2 h *(b+$ element widths $)$.

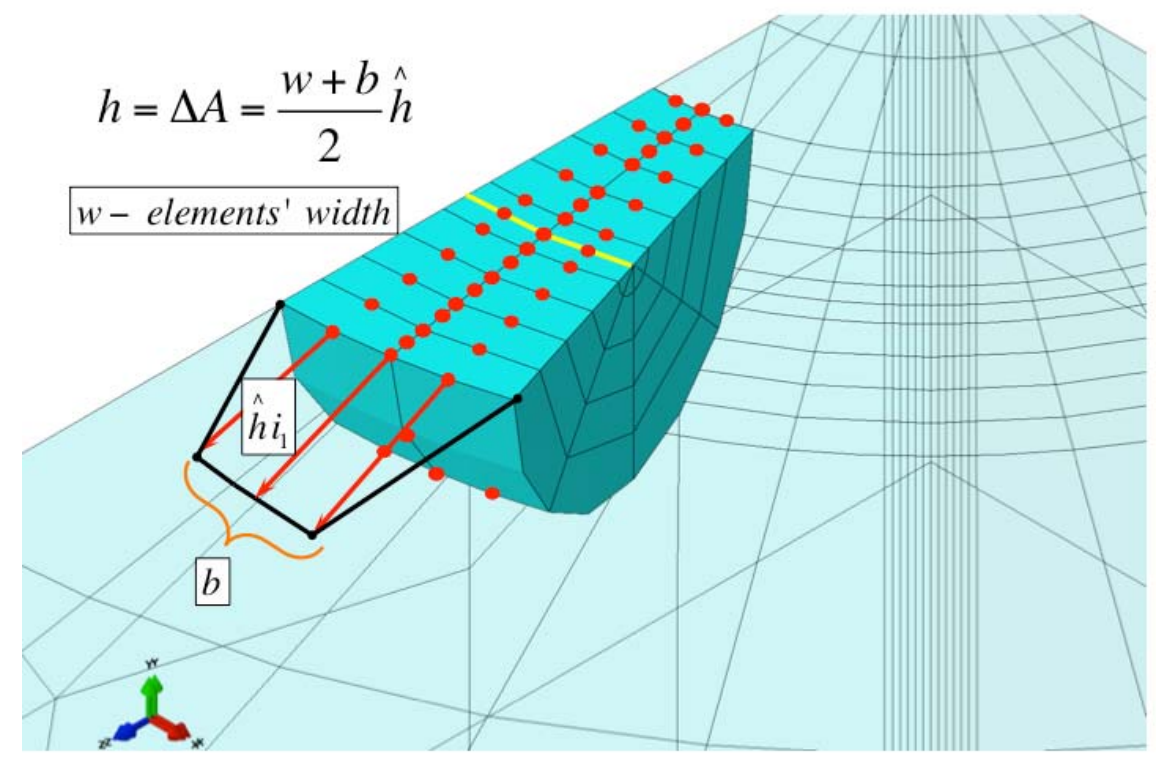

Figure 21 Trapezoidal localized perturbation 
Figure 22 shows a rectangular perturbation. All of the nodes in red were perturbed by a step size of $h=1 E-10$. No other nodes were perturbed. The area of the virtual crack extension was equal to $\Delta A=h *($ element widths $)$

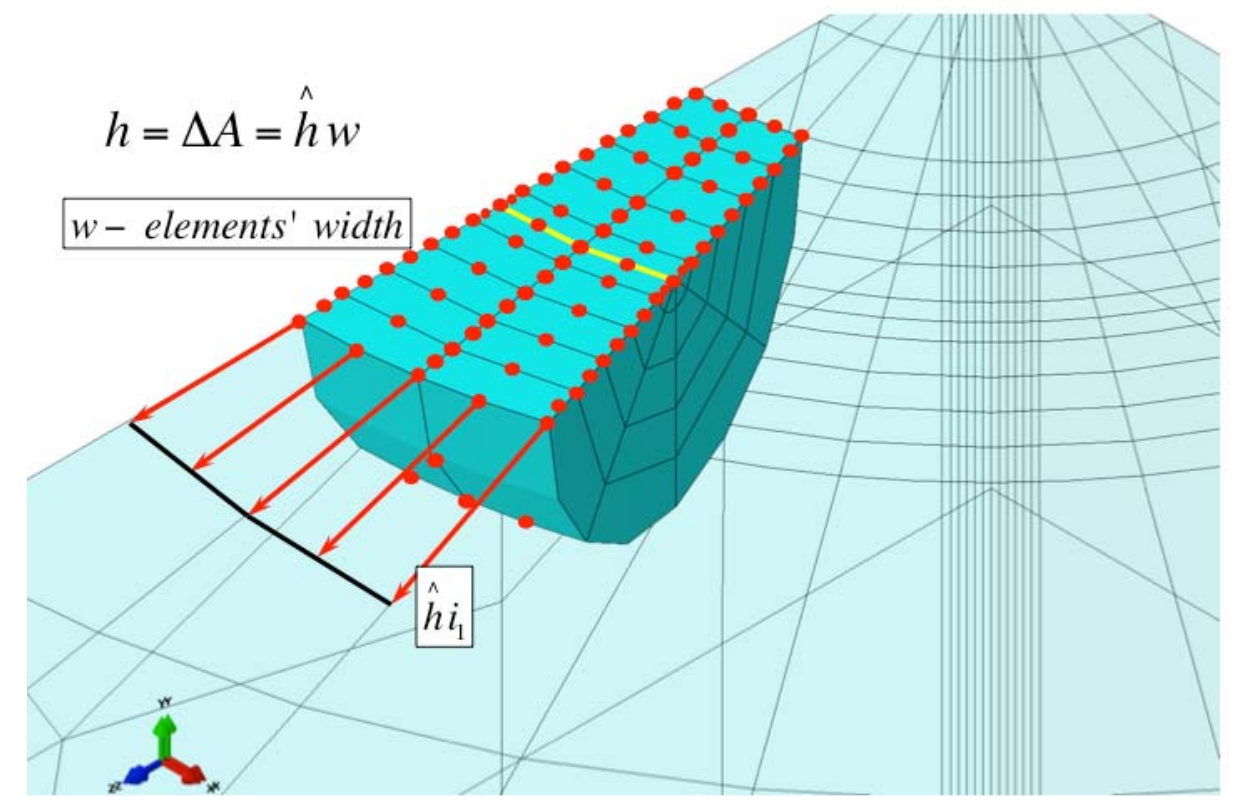

Figure 22 Rectangular localized perturbation

A comparison of the results of the energy release rate values relative to the analytical solution for each localized perturbation method are shown in Table 3. The results indicate that a local perturbation can be used to accurately compute a local value of the energy release rate along the crack front using either the triangular or rectangular perturbations; the trapezoidal perturbation was not accurate. The trapezoidal perturbation provides an abrupt change in perturbation within an element. A smooth transition should be provided as in the triangular case or the abrupt transition should be enacted at the element edge. Of course in all these cases, improved results can be obtained with finer meshes.

\begin{tabular}{|l|l|}
\hline Perturbation Case & Results \\
\hline
\end{tabular}




\begin{tabular}{|c|c|}
\hline Triangular & $0.46 \%$ \\
\hline Trapezoidal & $4.99 \%$ \\
\hline Rectangular & $0.43 \%$ \\
\hline
\end{tabular}

Table 3 Comparison of local energy release rate calculations with the analytical result

\section{Discussion}

The use of a complex finite element method to compute the energy release rate has some characteristics that may be appealing. Foremost, the method is elegant in its simplicity; equivalent to two analyses with a perturbed crack extension. However, the step size issue so detrimental to finite difference analysis is now moot. In fact, the method is not sensitive to the crack size extension if it is sufficiently small. Typical values uses are $10^{-10}$ times the element size at the crack tip or smaller.

Perhaps most important is that no additional equations are required in ZFEM to compute the energy release rate, as required for the $\mathrm{J}$ integral, or the stiffness derivative method, or the variational approach. If the physics of the phenomenon are contained with the finite element formulation, then ZFEM will be able to compute sensitivities with no additional effort besides the systemic "complexification" of the code. This means that the sensitivity capability essentially arrives as a byproduct of the analysis. As examples, no special provisions are needed for crack face loading, nor thermal loads, nor body forces, etc.

Second, the method is very general. Once a code is complexified, any shape sensitivity, material sensitivity, or loading sensitivity is available. Hence computing the energy release rate is an instance of a general shape sensitivity capability. This means that the evaluation of the energy release rate with respect to any variation in the crack extension is possible. Therefore, examination of kinked cracks, out of plane crack progressions, etc. can be explored easily simply through the appropriate input file specification of the imaginary coordinates. 
Third, results to date indicate that the computed energy release rate values are highly accurate; equivalent in accuracy to $\mathrm{J}$ integral calculations. In addition, the results are robust to the size of perturbation zone used for the virtual crack extension and the magnitude of the perturbation (step size) as long as the step size is sufficiently small. Empirical results indicate that perturbing a reasonably large group of nodes provides the best results. Suggested practice is to perturb a region containing 4 or 5 elements from the crack tip or larger.

Fourth, ZFEM provides the full field derivatives with respect to an extension in crack area. For example, the derivatives of the full displacement field and all post-processing quantities such as, strain, stress and strain energy are available from a single analysis. Hence the sensitivity results at all locations for all finite element quantities are available. A particular case in point is the development of weight functions that require the derivative of the crack opening displacement with respect to an extension in crack length along the crack line. These derivatives are available from a single ZFEM analysis.

Fifth, although not studied extensively, the method appears to be robust to the element discretization. For example, a "spider-web" rosette around the crack tip is not required to obtain accurate results. That is, ZFEM does not require a structured mesh that is often required for $J$-integral evaluations.

The price to pay for the complex finite element method is in run time and RAM requirements. This increase in computational time is due to the increase in the degrees-of-freedom of a complex analysis versus an all-real analysis. Typical run time comparisons indicate that ZFEM runs are between 3-4 times longer than equivalent real-variable runs. In addition, an important point to consider is that each complex analysis provides the derivative with respect to one crack extension. Hence, multiple complex analyses are required to compute multiple energy release rate solutions along a 3D crack front. 


\section{Conclusions}

The complex Taylor series expansion approach embodied within a complex variable finite element code was shown to be an effective method to compute the energy release rate using a number of example problems. The numerical accuracy in the computation of the energy release rate for all problems to date is equivalent to the J Integral.

The implementation of a complex-valued finite element method is straightforward as the traditional finite element formulation can be used, albeit with complex nodal coordinates and a systemic transformation of the code to complex variables. Computational times for a ZFEM analysis are typically 3-4 times larger than for the equivalent real-valued analysis. The ZFEM formulation demonstrated in this paper was implemented as a user element within the Abaqus commercial finite element code.

The ability to determine the crack perturbation through the setting of the imaginary nodal coordinates in the input file allows the analyst to easily control the crack extension. Hence, any crack perturbation including non-self-similar crack growth can be enacted simply through the appropriate setting of the imaginary coordinates. More generally, the virtual crack extension is a subset of the general shape sensitivity available with the complex finite element method. In addition, material and load sensitivity analyses are possible.

The beauty of ZFEM is that no additional equations need to be implemented in order to obtain the energy release rate, or any other sensitivity. If the physical phenomenon is embodied within the finite element formulation, then the energy release rate can be computed using ZFEM in a straightforward manner.

\section{Acknowledgements}

This work is a result of grants from the Army Research Office, grant no. W911NF1110208, and the Office of Naval Research, award no. N00014-14-1-0004. Special thanks are due to Patrick Golden, Material Directorate, Air Force Research Lab, Wright Patterson AFB, Dayton OH, and Dr. Manuel Garcia, Eafit University, Medellin Colombia for many helpful discussions and support. 


\section{References}

Abaqus finite element software, Dassault Systèmes Simulia Corp., Providence, RI, USA, 2015.

Anderson, T. L., "Fracture Mechanics: Fundamentals and Applications,” CRC Press, 1991.

Anderson, G. P., V. L. Ruggles, and G. S. Stibor. "Use of finite element computer programs in fracture mechanics." International Journal of Fracture Mechanics 7.1 (1971): 63-76.

Anderson, W. K., Newman, J. C., Whitfield, D. L., \& Nielsen, E. J., Sensitivity analysis for NavierStokes equations on unstructured meshes using complex variables, AIAA Journal 39 (2001) 56-63.

Banks-Sills, L., and D. Sherman. "On the computation of stress intensity factors for threedimensional geometries by means of the stiffness derivative and J-integral methods." International Journal of Fracture 53.1 (1992): 1-20.

Barbero, E. J., and J. N. Reddy. "The Jacobian derivative method for three $\square$ dimensional fracture mechanics." Communications in Applied Numerical Methods 6.7 (1990): 507-518.

Carpenter, W. C., D. T. Read, and R. H. Dodds Jr. "Comparison of several path independent integrals including plasticity effects." International Journal of Fracture 31, no. 4 (1986): 303-323.

DeLorenzi, Horst G. "Energy release rate calculations by the finite element method." Engineering Fracture Mechanics 21.1 (1985): 129-143.

DeLorenzi, H. G. "On the energy release rate and the J-integral for 3-D crack configurations." International Journal of Fracture 19 (1982): 183-193.

Garza, J. and H. R. Millwater, "Multicomplex Newmark-Beta Time Integration Method for Sensitivity Analysis in Structural Dynamics", AIAA Journal, Vol. 53, No. 5 (2015), pp. 1188- 1198, doi: http://arc.aiaa.org/doi/abs/10.2514/1.J0532821

Giner, E., F. J. Fuenmayor, A. J. Besa, and M. Tur. "An implementation of the stiffness derivative method as a discrete analytical sensitivity analysis and its application to mixed mode in LEFM." Engineering fracture mechanics 69, no. 18 (2002): 2051-2071.

Gomez-Farias, A., A. Montoya, H.R. Millwater, “Complex finite element sensitivity method for creep analysis," International Journal of Pressure Vessels and Piping (2015), http://dx.doi.org/10.1016/j.ijpvp.2015.05.006

Haber, R.B., and H.M. Koh. "Explicit expressions for energy release rates using virtual crack extensions." International Journal for Numerical Methods in Engineering 21, no. 2 (1985): 301-315.

Hellen, T. K., “On the Method of Virtual Crack Extensions.” International Journal for Numerical Methods in Engineering, Vol. 9, 1975, pp. 187-207.

Hwang, C. G., P. A. Wawrzynek, A. K. Tayebi, and A. R. Ingraffea. "On the virtual crack extension method for calculation of the rates of energy release rate." Engineering Fracture Mechanics 59, no. 4 (1998): 521-542.

Ingraffea, A.R. and P.A. Wawrzynek, Comprehensive Structural Integrity, Fracture of Materials from Nano to Macro, Volume 3: Numerical and Computational Methods, 3.01 "Finite Element Methods for Linear Elastic Fracture Mechanics,”, Elsevier Ltd., Oxford, UK, 2003

Lin, S.-C., and J.F. Abel. "Variational approach for a new direct-integration form of the virtual crack extension method." International Journal of Fracture 38.3 (1988): 217-235. 
Jing, Z., and X. R. Wu. "Wide-range weight functions and stress intensity factors for arbitrarily shaped crack geometries using complex Taylor series expansion method." Engineering Fracture Mechanics 138 (2015): 215-232.

Kim, S., J. Ryu, and M. Cho, (2011). Numerically generated tangent stiffness matrices using the complex variable derivative method for nonlinear structural analysis. Computer Methods in Applied Mechanics and Engineering, 200(1), 403-413.

Lei, Y., N.P. O'dowd, and G.A. Webster. "Fracture mechanics analysis of a crack in a residual stress field." International Journal of Fracture 106, no. 3 (2000): 195-216.

Millwater, H.R., D. Wagner, A. Baines, K. Lovelady, "Improved WCTSE method for the generation of 2D weight functions through implementation into a commercial finite element code," Engng Fract Mech, (2013), http://dx.doi.org/10.1016/j.engfracmech.2013.07.012

Montoya, A., R. Fielder, A. Gomez-Farias, and H.R. Millwater, "Finite Element Sensitivity for Plasticity using Complex Variable Methods," J. Eng. Mech. 1412 2014, DOI:10.1061/(ASCE)EM.1943-7889.0000837, 04014118.

Newman, J.C., D.L. Whitfield, and W.K. Anderson, Step-size independent approach for multidisciplinary sensitivity analysis, Journal of Aircraft 40 (2003) 566-573.

Nishioka, T., M. Kobashi, and S. N. Atluri. "Computational studies on path independent integrals for non-linear dynamic crack problems." Computational mechanics 3, no. 5 (1988): 331-342.

Parks, D. M., "A Stiffness Derivative Finite Element Technique for Determination of Crack Tip Stress Intensity Factors.” International Journal of Fracture, Vol. 10, issue 4, 1974, pp. 487-502.

Riedel, H. "Creep deformation at crack tips in elastic-viscoplastic solids." Journal of the Mechanics and Physics of Solids 29, no. 1 (1981): 35-49.

Sanford, R.J. Principles of fracture mechanics. Pearson College Division, 2003.

Shih, C.f., B. Moran and T. Nakamura, "Energy release rate along a three-dimensional crack front in a thermally stressed body", International Journal of Fracture 30, (1986) 79-102.

Squire, W., and G. Trapp, Using complex variables to estimate derivatives of real functions, Soc. Indus. Appl. Math. 40 (1998) 110-112

Suo, X.-Z., and A. Combescure. "Double virtual crack extension method for crack growth stability assessment." International journal of fracture 57.2 (1992): 127-150.

Szekrényes, A., and J. Uj. "Comparison of some improved solutions for mixed-mode composite delamination coupons." Composite Structures 72, no. 3 (2006): 321-329.

Tada, H., P.C. Paris, and G.R. Irwin, The Stress Analysis of Cracks Handbook, Del Research Corporation, Hellertown, Pennsylvania, 1973

Tracey, D.M. "Finite elements for determination of crack tip elastic stress intensity factors." Engineering Fracture Mechanics 3.3 (1971): 255-265.

Voorhees, A., H.R. Millwater, and R.L. Bagley, "Complex Variable Methods for Shape Sensitivity of Finite Element Models," Finite Elem. Anal. Des., 47 (2011) 1146-1156, doi:10.1016/j.finel.2011.05.003

Voorhees, A., H.R. Millwater, R. Bagley, and P. Golden, "Fatigue Sensitivity Analysis Using Complex Variable Methods," Int J Fatigue 40 (2012) 61-73, doi:10.1016/ j.ijfatigue.2012.01.016 
Wagner, D. and H.R. Millwater, "2D Weight Function Development using a Complex Taylor Series Expansion Method," Engng Fract Mech 86 (2012), 23-37, 210-

doi:10.1016/j.engfracmech.2012.02.006

Waisman, H., "An analytical stiffness derivative extended finite element technique for extraction of crack tip strain energy release rates." Engineering Fracture Mechanics 77.16 (2010): 3204-3215.

Wang, B.P., and A.P. Apte, Complex variable method for eigensolution sensitivity analysis, AIAA Journal. 44 (2006), 2958-2961

Watwood, V. B. "The finite element method for prediction of crack behavior." Nuclear Engineering and Design 11.2 (1970): 323-332.

Wilson, W. K., and I-W. Yu. "The use of the J-integral in thermal stress crack problems." International Journal of Fracture 15, no. 4 (1979): 377-387.

Yang, Z.J., J.F. Chen, and G.D. Holt. "Efficient evaluation of stress intensity factors using virtual crack extension technique." Computers \& Structures 79.31 (2001): 2705-2715.

Zhao, X. C., X. R. Wu, and D. H. Tong. "Weight functions and stress intensity factors for pin loaded single edge notch bend specimen." Fatigue \& Fracture of Engineering Materials \& Structures (2015). 